\title{
Carácter y hábitos para el aprendizaje: definición y proyecto de medición
}

\section{Character and learning habits: definition and measurement proposal}

\author{
Dr. Ignasi de BOFARULL. Profesor Contratado Doctor. Universitat Internacional de Catalunya (ibofarul|@uic.es).
}

\section{Resumen:}

Este trabajo estudia cómo influye el carácter, los hábitos y la actitud del estudiante en los procesos de enseñanza-aprendizaje. Hasta hace poco la educación del carácter constituía un asunto de educación moral y cívica. Ahora, las nuevas investigaciones sobre las habilidades no-cognitivas y el aprendizaje socioemocional reflejan cómo estos planos de la personalidad dan consistencia a los procesos de enseñanza-aprendizaje escolares. Las neurociencias subrayan aquí el valor de las funciones ejecutivas: la atención, la tenacidad y la planificación son momentos donde el estudiante despliega su aprendizaje. La inteligencia clásica (cociente intelectual, CI) pone el acento en la comprensión analítica, un momento puntual; la inteligencia del carácter pone el foco en los procesos volitivos que fraguan el trabajo intelectual que empieza en el aula y acaba en la planificación del estudio en el hogar. Una vez definidas las habilidades no-cognitivas, las funciones ejecutivas y el carácter, marcos emparentados entre sí y presentes en la vida escolar y familiar, el segundo objetivo ha sido valorar cómo el am- biente socio-familiar interviene agudamente en estos procesos. El tercer objetivo ha sido, siempre en paralelo con el despliegue del estudio, proponer las herramientas que midan estas fortalezas en la educación primaria: BFQ-N y BRIEF 2. Si se proponen modelos de formación del carácter para el aprendizaje se deben ofrecer herramientas para su medición orientadas a testar el éxito de estos programas. La conclusión apunta a una iniciativa de calado: la universidad, la escuela y los agentes educativos deben pensar un nuevo modelo de educación integral de sus estudiantes desde esta convergencia entre carácter e inteligencia clásica. El fracaso y el abandono escolar tienen razones académicas, pero también familiares y personales. Este tercer milenio, complejo, cambiante, necesita habilidades robustas y flexibles para hacer frente a los retos de una sociedad que no nos desvela aún a dónde va.

Descriptores: carácter, hábitos, habilidades no-cognitivas, funciones ejecutivas, aprendizaje socio-emocional, apego, actitud, educación integral, habilidades para el siglo xxI.

Fecha de recepción de la versión definitiva de este artículo: 30-03-2018.

Cómo citar este artículo: De Bofarull, I. (2019). Carácter y hábitos para el aprendizaje: definición y proyecto de medición | Character and learning habits: definition and measurement proposal. Revista Española de Pedagogía, 77 (272), 47-65. doi: https://doi.org/10.22550/REP77-1-2019-03 


\section{Abstract:}

This work examines how students' character, habits, and mindset influence teaching-learning processes. Until recently character education was a matter of moral and civic education, but recent research into non-cognitive skills and social-emotional learning reflects how these personality traits give steadiness to school teaching-learning processes. Neuroscience here emphasises the value of executive functions: attention, inhibitory control, and planning are moments where the student unfolds her learning. Classical intelligence (IQ) focuses on analytical understanding, a specific moment; character intelligence focuses on the volitional processes that create the intellectual work that begins in the classroom and ends with planning of study at home. The first objective is to define non-cognitive skills, executive functions, and character, related frameworks that are present in school and family life. After this, the second objective is to assess how the social-family environment affects these processes. The third objective, in parallel with carrying out the study, is to propose tools to measure these strengths in elementary school: BFQ-N and BRIEF 2. If character education models for learning are proposed, tools should be offered to measure it intended to test the success of the programmes. The conclusion identifies a major initiative: universities, schools, and educational agents should think about a new integrated model of education for their students based on this convergence between character and classical intelligence. School failure and dropout have academic explanations but also family and personal ones. This complex and changing third millennium requires robust and flexible skills to face the challenges of a society that has still not shown us where it is going.

Keywords: character, habits, non-cognitive skills, executive functions, social and emotional learning, attachment, mind-set, whole education, 21st century skills.

\section{Habilidades de carácter}

Cuando un niño nace presenta un temperamento marcado biológicamente que, a lo largo de los años, se va a ir configurando en un carácter que será el producto de unos hábitos adquiridos y mediados por el entorno familiar, escolar y social. El objetivo escolar, a nuestro entender, es que los estudiantes cuenten con un carácter que acabe siendo la suma de unos hábitos alcanzados en repetidos actos de una voluntad de índole cognitiva, ética y comportamental. Y enmarcamos este objetivo en los últimos cursos de la educación infantil y en toda la educación primaria. Estamos hablando de un aprendizaje de hábitos que debe arraigar en el plano cerebral y neuronal. Como ya intuyó James (James, 1899; Alcover y Rodríguez, 2012) a finales del siglo XIx, los hábitos conformarán un nuevo aprendizaje-comportamiento más ágil y dúctil, gracias a la neuroplasticidad, es decir, a una modificación de las redes neuronales y de la estructura física del cerebro. El resultado será que el estudiante configurará una segunda naturaleza, un nuevo funcionamiento cognitivo y conductual capaz de asentar destrezas de pensamiento del más alto rango, que a su vez será capaz de seguir desarrollando a lo largo de su vida. 
Estos conceptos de hábito y de carácter están anclados en una tradición occidental que arranca en la filosofía de Aristóteles, sobre todo en su libro Ética a Nicómaco, (Malikail, 2003; Bernacer y Murillo, 2014) y que llegan hasta hoy con una vigencia muy alta. El entrenamiento de los hábitos como base del aprendizaje es una línea que conecta Aristóteles con las neurociencias, pasando por las intuiciones de James. El buen carácter, dicho sucintamente, se constituye sobre aquellos hábitos y fortalezas que nos permiten conducirnos cabalmente por la vida en la consecución de nuestras metas familiares, laborales y ciudadanas. Es más, una buena educación para la ciudadanía no debe olvidar que los ciudadanos comprometidos y participativos, críticos y capaces de construir una democracia de calidad deben ser educados en un buen carácter (Althof y Berkowitz, 2006). Una ciudadanía sin virtudes cívicas, hablando en términos aristotélicos, puede poner en riesgo la sostenibilidad de un modelo de sociedad. Estamos hablando pues de la educación integral (Gervilla, 2000).

La psicología de la personalidad describe estos procesos desde hace décadas. Nosotros nos planteamos definir y proponer una medición de cómo el carácter, los hábitos y las fortalezas personales inciden en el proceso de aprendizaje escolar. Y decimos proceso de aprendizaje pues este aprendizaje escolar es secuencial y se construye (o deteriora) no solo en el aula, sino también en los marcos familiares, sociales, durante el estudio y la planificación de las tareas, y en la actualidad, también compitiendo con la distractora actividad digital (redes sociales, etc.) (Chen y Yan, 2016).
Lo que está sucediendo hoy en la vida escolar es que a menudo nos olvidamos de cómo los resortes del carácter influyen en el aprendizaje. La Real Academia Española define carácter como: «fuerza y elevación de ánimo natural de alguien, firmeza, energía». El carácter es un motor personal que constantemente regula la secuencia cognición-deliberación-decisión-acción (Vigo, 2012). Y el momento de la deliberación-decisión no es solo un paso únicamente racional, sino que es una elección plagada de emociones, creencias y actitudes en curso (Damasio, 1996). En una palabra (y recuperando la vertiente pedagógica de James): escolarmente hay que formar a los estudiantes en la destreza de conocer-deliberar-decidir-actuar contando con las emociones, las creencias y las actitudes más inteligentes fundadas en unos hábitos arraigados y sabios que los maestros con tacto (tactful teacher) saben despertar (Thoilliez, 2013). En los Estados Unidos cuando se habla de la educación del carácter se cita la tríada cabeza, corazón, manos: head, heart, hands (Lickona, 1991). A los maestros y a los estudiantes lo mejor que les puede suceder es estar motivados por profundizar en una disciplina 0 en una tarea. $Y$ es menos interesante hacerlo a palo seco y solo en función del sentido del deber. Aunque a veces el sentido del deber sostendrá la pasión y la motivación, porque el objetivo final es profundamente ilusionante. Cada día, los maestros y profesores certifican que las fortalezas personales, los hábitos operativos positivos, las motivaciones intrínsecas y la autorregulación de los estudiantes se ve reflejada positivamente en sus calificaciones. Y lo contrario: la abulia, la desatención, la impulsividad son la antesala del fracaso 
escolar. En el plano más técnico se sabe que estas habilidades de carácter, desplegadas en diferentes planos de la personalidad, cuando convergen son, en parte, condiciones que posibilitan el mejor aprendizaje:

A lo largo de este artículo, usamos el término habilidades de carácter para describir los atributos personales que se considera que no son medibles por pruebas de CI (cociente intelectual) o pruebas de rendimiento. Estos atributos tienen muchos nombres en la literatura, incluyendo habilidades blandas (soft-skills), rasgos de personalidad, habilidades no-cognitivas, destrezas no cognitivas, carácter y habilidades socioemocionales. Estos diferentes nombres connotan diferentes propiedades (Heckman y Kautz, 2013, p. 3) ${ }^{1}$.

\section{Autorregulación y funciones ejecutivas}

Regresamos a la relación existente entre hábito, aprendizaje y neuroplasticidad. Las ciencias del neurodesarrollo lo subrayan con insistencia: la autorregulación es una parte vital del aprendizaje y, a la vez, con el paso de los años, es parte de una vida equilibrada y estable emocionalmente (Shonkoff, Boyce y McEwen, 2009). Esta autorregulación, también denominada control inhibitorio, es una capacidad fundamental para manejarse en casi todos los planos de la vida. Y es a la vez uno de los ejes de las funciones ejecutivas base de las habilidades de carácter (FE desde ahora) (Diamond, 2013; Diamond y Lee, 2011).

Unas FE que dependen en su funcionamiento del córtex prefrontal y cuyo primer y sensible desarrollo tiene lugar en los tres primeros años de vida (McEwen, 2016). Unas FE (memoria de trabajo, control inhibitorio y pensamiento-comportamiento flexible) (Diamond, 2013) que se podrían definir como un conjunto de competencias cognitivo-conductuales de orden superior que razonan y analizan los distintos contenidos escolares (y casi todas las realidades vitales) con el objetivo de tomar decisiones prácticas. Con frecuencia, estas decisiones se convierten, consecuentemente, en unas tareas que deben ser desplegadas en planes a corto, medio y largo plazo. Las FE siguen actuando también ahí: en la planificación y la consecución de los objetivos, tanto en la escuela como en la familia o en la vida (Diamond, 2014). Para la medición general del carácter y los hábitos para el aprendizaje proponemos una herramienta que evalúa globalmente la personalidad en estas edades escolares: es el BFQ-N (apartado 6). Se verá más adelante. Sin embargo, existe un cuestionario que mide más concretamente las FE con mucha precisión. Nos proponemos una investigación que evalúe las habilidades de carácter y que, en esa dirección, busque la triangulación desde diferentes perspectivas y, en consecuencia, trabaje desde convergentes instrumentos. Por tanto, para la medición particular de las FE proponemos el BRIEF 2 (Gioia, Isquith, Guy, y Kenworthy, 2000).

El BRIEF (del inglés, behavior rating inventory of executive function) es una escala compuesta por dos cuestionarios, uno para padres y otro para docentes, diseñados para evaluar las FE en el hogar y en la escuela, respectivamente (Soprano, 2003, p. 45) (ver Tabla 1). 
El BRIEF 2, en su validación española, también ofrece la perspectiva escolar unida a la familiar entre los 5 y los 18 . Y es que debemos recordar que en el presente trabajo, como he- mos señalado en las primeras líneas, buscamos la evaluación de las $\mathrm{FE}$ en el final de la educación infantil y, sobre todo, en la educación primaria (Maldonado-Belmonte, 2016).

TABLA 1. Áreas de las funciones ejecutivas exploradas por la escala BRIEF.

\begin{tabular}{|c|c|}
\hline Inhibición & $\begin{array}{l}\text { Habilidad para resistir a los impulsos y detener una conducta en el mo- } \\
\text { mento apropiado. }\end{array}$ \\
\hline & $\begin{array}{l}\text { Habilidad para hacer transiciones y tolerar cambios, flexibilidad para resolver } \\
\text { problemas y pasar el foco atencional de un tema a otro cuando se requiera. }\end{array}$ \\
\hline $\begin{array}{c}\text { Control } \\
\text { emocional }\end{array}$ & Refleja la influencia de las FE en la expresión y regulación de las emociones. \\
\hline Iniciativa & $\begin{array}{l}\text { Habilidad para iniciar una tarea o actividad sin ser incitado a ello. Incluye } \\
\text { aspectos tales como la habilidad de generar ideas, respuestas o estrategias } \\
\text { de resolución de problemas de modo independiente. }\end{array}$ \\
\hline $\begin{array}{l}\text { Memoria } \\
\text { de trabajo }\end{array}$ & $\begin{array}{l}\text { Capacidad para mantener información en la mente con el objeto de completar } \\
\text { una tarea, registrar y almacenar información o generar objetivos. La memoria } \\
\text { de trabajo sería esencial para llevar a cabo actividades múltiples o simultáneas, } \\
\text { como puede ser el caso de cálculos aritméticos, o seguir instrucciones complejas. }\end{array}$ \\
\hline $\begin{array}{l}\text { Organización } \\
\text { y } \\
\text { planificación }\end{array}$ & $\begin{array}{l}\text { Son componentes importantes para la resolución de problemas. Organiza- } \\
\text { ción implica la habilidad para ordenar la información e identificar las ideas } \\
\text { principales o los conceptos clave en tareas de aprendizaje o cuando se trata } \\
\text { de comunicar información, ya sea por vía oral o escrita. Planificación invo- } \\
\text { lucra plantearse un objetivo y determinar la mejor vía para alcanzarlo, con } \\
\text { frecuencia a través de una serie de pasos adecuadamente secuenciados. }\end{array}$ \\
\hline Orden & $\begin{array}{l}\text { Otro aspecto de la organización es la habilidad para ordenar las cosas del } \\
\text { entorno, e incluye mantener el orden en los elementos de trabajo, jugue- } \\
\text { tes, armarios, escritorios u otros lugares donde se guardan cosas, además } \\
\text { de tener la certeza de que los materiales que se necesitarán para realizar } \\
\text { una tarea estén efectivamente disponibles. }\end{array}$ \\
\hline $\begin{array}{l}\text { Control } \\
\text { (monitoring) }\end{array}$ & $\begin{array}{l}\text { Comprende dos aspectos: } \\
\text { a. El primero se refiere al hábito de controlar el propio rendimiento durante la } \\
\text { realización de una tarea o inmediatamente tras finalizar la misma, con el objeto } \\
\text { de cerciorarse de que la meta propuesta se haya alcanzado apropiadamente. } \\
\text { b. El segundo, que los autores llaman autocontrol (self-monitoring), refleja la } \\
\text { conciencia del niño acerca de los efectos que su conducta provoca en los demás. }\end{array}$ \\
\hline
\end{tabular}

Fuente: Elaboración propia a partir de Soprano, 2003, p. 45.

\section{Habilidades cognitivas, habi- lidades no-cognitivas y su medi- ción}

Repasada la literatura sobre las habilidades de carácter, adentrémonos en su medición. De todos es sabido que el aprendizaje escolar no solo se ciñe a un currículum prefijado; no solo consiste en la incorporación analítica y cognitiva de los contenidos de competencias, materias, y objetivos por parte de los estudiantes. La función de la escuela no solo se reduce a la obtención de unas calificaciones frías que consigue cada estudiante y que se concretan en la evaluación de sus maestros donde se certifica el aprendizaje de los contenidos curriculares. Este aprendizaje y esta inteligencia, entendida en sentido clásico, se miden por los innumerables test existentes que consignan lo que conocemos como el Cociente Inte- 
lectual (CI). Pero no solo existe esta inteligencia cognitiva estrictamente académica. Existen además unas habilidades no-cognitivas - la personalidad, el carácterque fundamentan y apuntalan la misma inteligencia académica, conformando una nueva inteligencia más global. Hablar de habilidades no-cognitivas como base de la inteligencia académica parece una contradicción. Los rasgos de la personalidad y del carácter que intervienen en las habilidades no-cognitivas también son cognición, aunque lo hacen en su vertiente más volitiva y comportamental. Sin embargo, este nombre capta todo aquello que no entra dentro de lo que se denomina estrictamente habilidades cognitivas: "hablar de habilidades no-cognitivas puede inducir a error, pues parece un nombre equivocado. Cada proceso psicológico es cognitivo en el sentido en que supone algún tipo de procesamiento de información»(West et al., 2016, p. 149)².

En el mundo anglosajón, a estas habilidades las llaman también habilidades dúctiles (soft-skills) dada su maleabilidad. Estamos ante unas habilidades que son, consecuentemente, moldeables, no fijas. Unas capacidades educables que se pueden cultivar y medir a través de distintos instrumentos. Estamos pues ante dos tipos de habilidades -también podríamos llamarlas inteligencias-, que están en la base del aprendizaje. Las primeras son más cognitivas, analíticas y comprensivas (CI, IQ), dicho sucintamente. Mientras que las segundas se mueven más allá de lo que siempre se ha entendido por las parcelas cognitivas y promueven buenos resultados en el aprendizaje a través de la mejora de hábitos. Hábitos y actitudes, como la per- severancia, la concentración, la focalización en el estudio, sumado a unas creencias positivas en la propia capacidad, que tienen un gran recorrido motivacional. Y no estamos ante una nueva inteligencia no-solo-cognitiva, solo teorizada, sino que estamos ante un nuevo modo de entender la inteligencia que es observable y que se mide en términos de rasgos de la personalidad y del carácter.

Estos rasgos son cuantificables desde hace tiempo a partir de los 17 años y uno de los instrumentos más destacables - entre otros - es el cuestionario de la personalidad Big Five (BFQ) (John y Srivastava, 1999). En cualquier caso, nuestro interés es el aprendizaje y el estudio de la inteligencia de carácter y socio-emocional al final de la escolarización infantil y, sobre todo, en Primaria (5-12 años). Para este fin, contamos con una versión del Big Five (BFQ) en español para estudiantes de 8 a 17 años. Se trata del BFQ-N: un cuestionario que presenta los requisitos psicométricos establecidos cuando los informantes son los propios niños (Carrasco-Ortiz, Holgado-Tello y Del Barrio-Gandara, 2005). En el sexto apartado profundizaremos en los factores y las facetas que mide el $\mathrm{BFQ}$.

\section{Entorno socio-familiar, rendi- miento escolar y brecha educativa}

Estos procesos escolares de enseñanza-aprendizaje a menudo se construyen sobre unas habilidades y rasgos de la personalidad que preceden a la escuela y continúan paralelamente durante la escolarización. Su raíz está en la herencia y continúa en el ambiente familiar y so- 
cial (Bronfenbrenner, 2009). Un ambiente en el que destaca el papel de los padres y cuidadores más cercanos (Moullin, Waldfogel y Washbrook, 2014). Si los entornos familiares son precarios, estresantes y caóticos estas habilidades de carácter pueden quedar mermadas (Perry, 2009), por ejemplo, en el plano de la impulsividad y de las dificultades en la atención. $Y$ a la inversa: los hogares mejor organizados, afectivos y más previsibles son los que facilitan el aprendizaje de los futuros estudiantes desde los primeros meses de vida. Estas familias, gracias a su orden, su predictibilidad, su coherencia y sus hábitos saludables facilitan la adquisición de rutinas y habilidades con alto rendimiento académico (Hanscombe, Haworth, Davis, Jaffee y Plomin, 2011). Sin embargo, estos estudios no nos deben hacer pensar que los altos ingresos son la variable primaria que facilita la emergencia de habilidades de carácter. Puede suceder que la calidad de las interacciones, el orden, la coherencia en el hogar esté presente en las disposiciones de unas familias de un estatus económico no necesariamente alto, sino medio o medio-bajo (Tough, 2014 y 2016). Y paralelamente, podría haber hogares de altos ingresos y a la vez tan caóticos que el aprendizaje se hace difícil, entre otras razones, porque el cultivo de las funciones ejecutivas ha quedado deteriorado (Vernon-Feagans, et al., 2016). El clima y aprendizaje familiar en los años preescolares y escolares, está marcado por variadas perspectivas psicológicas y marcos conceptuales. Veremos los que consideramos más relevantes. Un ejemplo claro de esta raíz socio-familiar que puede condicionar el aprendizaje es el vínculo del apego que está situado en el núcleo de la parentalidad (en las interacciones progenitor-infante) y se configura en los dos primeros años de vida. En cualquier caso, su sutil y profunda realidad continúa estando presente a lo largo del ciclo vital de cada persona. Galán-Rodríguez nos ofrece una definición muy incisiva de apego:

Bowlby propuso una concepción relacional del ser humano (el niño viene al mundo preparado para establecer un estrecho lazo de unión con una figura de cuidado) sostenida por un marco conceptual de gran interés. Las aportaciones de Ainsworth permitieron la expansión (conceptual y académica) de la Teoría del Apego, consolidando así lo que parecía una fructífera y prometedora línea de trabajo (Galán-Rodríguez, 2010, p. 581).

Este vínculo con la madre, o la figura de apego, puede llegar a ser decisiva y va a fijar la personalidad segura 0 insegura del futuro estudiante (Bowlby, 1969; Ainsworth y Marvin, 1995). Una seguridad y una confianza que el niño adquirirá en sí mismo y en el mundo y que va a ser muy valiosa cuando se inicia la escolarización.

Otro marco conceptual es el estilo lingüístico y atributivo que genera el entorno familiar y social que rodea al infante y que puede ser propositivo 0, a la inversa, desalentador. Estamos hablando de los códigos lingüísticos (elaborados o restringidos) que el niño aprende en casa (Bernstein, 1989) y en sus entornos más cercanos. Unos códigos que luego se despliegan -positiva o negativamente- de un modo imperceptible pero constante en la propia escuela. 
Ahí nace la brecha educativa. La carencia de estos factores (National Academies of Sciences, Engineering, and Medicine, 2016, p. 81) puede ser determinante:

- Vida saludable (nutrición, higiene, sueño).

- Apego seguro.

- Orden, coherencia en el hogar, predictibilidad.

- Límites y disciplina cálida.

- Riqueza en el intercambio del lenguaje.

- Sosiego y disponibilidad familiar en las interacciones con adultos.

- Sensibilidad educativa, cultural, lectora.

Solo un par de ejemplos para ilustrar las afirmaciones anteriores: frecuentar con los hijos, desde muy temprano, las bibliotecas municipales, el juego compartido en el hogar (no digital) (Shaheen, 2014), las excursiones donde la naturaleza es protagonista y creer y apoyar a la escuela desde el hogar (Tough, 2016) está al alcance de cualquier bolsillo.

Consecuentemente, luchar contra la brecha educativa pasa por (Center on the Developing Child at Harvard University, 2017) (ver Gráfico 1):

a) Formar bien a las familias en asuntos como las interacciones padres-hijos.

b) El reforzamiento de las habilidades centrales para la vida (entre las cuales están las FE).

c) La reducción del estrés en las vidas de los niños y las familias.

La escuela tiene un papel fundamental pero el fracaso escolar empieza imperceptiblemente en casa en los primeros meses de vida. Recordemos una vez más que hay familias interesadas en temas educativos con bajos ingresos, y familias con altos ingresos con muy poca sensibilidad en estos planos. Más que altos ingresos lo que precisa un hogar es estabilidad económica. Y, para algunos estudiosos, más sobriedad que opulencia en el consumo diario. La brecha socio-educativa está más centrada en un clima familiar propositivo que en los ingresos y los recursos, como se certifica en el Gráfico 1. En cualquier caso, si los recursos materiales caen por debajo de un cierto umbral de pobreza, no hay clima familiar que supere esta adversidad. Las carencias materiales severas son entonces un estresor muy influyente.

Bourdieu (1986), sociólogo de la educación, ha estudiado estos condicionamientos estructurales y estructurantes con detenimiento desde su concepto de habitus. Y las habilidades no-cognitivas se inscriben en este gran magma que es el habitus fijado en el origen social. Es un concepto mucho más complejo que el que entendemos por hábito. Son percepciones, sensibilidades, inclinaciones estéticas y cultas. El ocio (Palmero-Cámara et al., 2015; De Bofarull, 2005) de las clases privilegiadas también incide en el habitus. El habitus radica en una cultura que se traduce en acciones, elecciones y disposiciones ilustradas. Uno de los estudios de Bourdieu se titula así: La distinction. Critique sociale du jugement (1979). Sintetizando un poco se podría decir que hay un habitus socio-familiar idóneo para integrar los conocimientos escolares que está presente normalmente en familias de alta cultura e ingresos. 
GRÁFICo 1. Tres principios para la mejora de los resultados en niños y familias. Política y práctica.

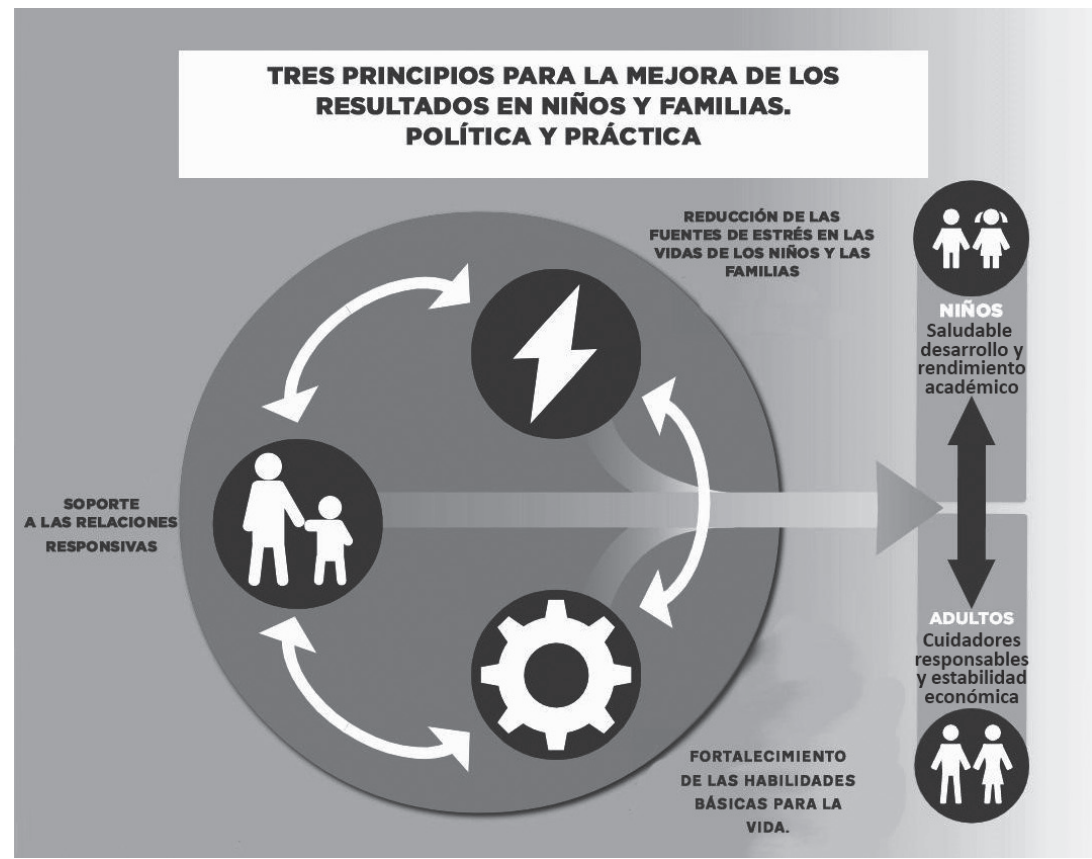

Fuente: Center on the Developing Child at Harvard University, 2017.

En el otro extremo se da un habitus limitado, inclinado a cierta vulgaridad, que se halla en familias de poca cultura e ingresos que por su llaneza y carencias no va a facilitar la integración de los conocimientos escolares por parte de sus hijos. El idioma de los contenidos y las materias de la escuela les resulta a menudo incomprensible y lejano para estas últimas familias. Los niños que proceden de un refinado habitus entienden perfectamente el idioma de la escuela.

A veces, la escuela infantil y primaria trabaja la mejora de estas condiciones subyacentes - habitus lingüístico y cultural restringido, apego - en el despliegue de los procesos de enseñanza-aprendizaje, aunque a menudo le resulta muy difícil revertirlos si están arraigados. Suele suceder, insistimos, que los estudiantes a menudo carecen de estas habilidades básicas y la escuela obligatoria las da por supuestas (Kautz, Heckman, Diris, Ter Weel y Borghans, 2014). Ahí radican algunas variables del fracaso y el abandono escolar: en las actitudes interiores, en las creencias, en las percepciones, en la implicación de las familias con los niños más pequeños. Los influyentes informes PISA, a partir de 2012, han empezado a plantearse la existencia de estas habilidades como parte de su tarea de evaluación de los estudiantes de 15 años en los países de la OCDE: Students' Engagement, Drive and Self-Beliefs (OCDE, 2013). Y quizá es el primer paso para que diferentes países tengan en cuenta al estudiante no como un ser abstracto que aprende, sino como una persona compleja, diversa, con muy distintas necesidades y procedencias en diversos planos: cognitivo, psicológico, social y ma- 
terial. Unas necesidades materiales que, a menudo, se unen a ciertas adversidades agudas y estresantes (paro, trabajo precario, enfermedad, monoparentalidad, etc.) que se han multiplicado en los últimos años dada la inestabilidad — política, económica, laboral, familiar - que se está viviendo en Occidente desde las crisis de 2008.

\section{Aprendizaje socioemocional y autorregulación}

En esta dirección, la escuela, desde hace unos años, está abogando por la educación en valores capaces de superar los déficits en los planos social, cultural, educativo $\mathrm{y}$ emocional que el estudiante concreto trae de su ambiente socio-familiar y que pueden suponer rémoras en el desarrollo académico. En los últimos años esta educación en valores se ha desarrollado a partir de un marco sólido de procedencia anglosajona: estamos hablando del aprendizaje socioemocional (SEL: social and emotional learning). El aprendizaje socioemocional (Durlak, Dymnicki, Taylor, Weissberg y Schellinger, 2011; Álvarez-Hevia, 2018) es otro término paraguas que engloba unas habilidades que están más allá, de nuevo, de la inteligencia clásica y que contribuyen a poner las bases del aprendizaje desde distintos rasgos de la personalidad.

El aprendizaje socio-emocional es un buen avance escolar en la educación moral, contribuye a mejorar el clima de la clase y la escuela, y también pueden medirse sus resultados desde el más arriba mencionado cuestionario Big Five (John y Srivatava, 1999). El aprendizaje socioemocional se organiza en cinco competencias centrales:
1) Autoevaluación (self-awareness).

2) Autogestión (self-management).

3) Conciencia social (social awareness).

4) Habilidades relacionales (relationship skills).

5) Toma de decisiones responsable (responsible decision-making).

Los resultados están siendo muy efectivos en los Estados Unidos y cuentan con unos retornos considerables (1:11 por dólar invertido) en la relación costes-beneficios cuando se aplican estos programas en el ámbito escolar (Elias et al., 2015). Sin embargo, es muy importante dejar constancia de que este marco conceptual, en su aplicación, exige un encaje sistémico donde juegan un papel interdependiente: a) el hogar y la comunidad; b) la escuela; y c) el aula. Solo en la convergencia de estos agentes educativos es posible el desarrollo del carácter, de los hábitos, de la autorregulación. Dicho en otras palabras: solo desde estas competencias crece un aprendizaje socio-emocional sosegado, oportuno y organizado (ver Tabla 2 y Gráfico 2). Permitamos que se definan ellos mismos y certificaremos los puntos en común con los objetivos del presente estudio:

El aprendizaje socioemocional (SEL) es el proceso mediante el cual niños y adultos adquieren y aplican acertadamente el conocimiento, las actitudes y las habilidades necesarias para comprender y manejar las emociones, establecer y alcanzar metas positivas, sentir y mostrar empatía por los demás, establecer y mantener relaciones positivas y tomar decisiones responsables (CASEL, 2017, p. 3). 
Esta inteligencia emocional va a ser y éticas decisiones en la escuela y en la base también de las más reflexivas la vida.

TABLA 2. Marco conceptual para un aprendizaje social y emocional sistémico.

\section{Hogares y comunidad asociados con la escuela $+$ \\ Escuelas con prácticas SEL en todos los ámbitos $+$}

Aulas en las que SEL se aplica curricularmente
Identificar las emociones.

Autopercepción precisa.

Reconocimientos de las fortalezas.

Confianza en uno mismo.

Conciencia de las propias capacidades.

Control del impulso.

Gestión del estrés.

Autodisciplina.

Automotivación.

Establecimiento de metas.

Habilidades organizativas.

Identificación de los problemas.

Análisis de las situaciones.

Responsable toma de decisiones

Resolución de problemas.

Evaluación.

Reflexión.

Responsabilidad ética.

Comunicación.

Compromiso social.

Habilidades

Establecimiento de relaciones.

Trabajo en equipo.

Toma de perspectiva.

Empatía.

Conciencia social

Reconocimiento de la diversidad.

Respeto por los otros.

Fuente: Elaboración propia a partir de Casel, 2017, p. 3. 


\section{GRÁFICo 2. Aprendizaje social y emocional}

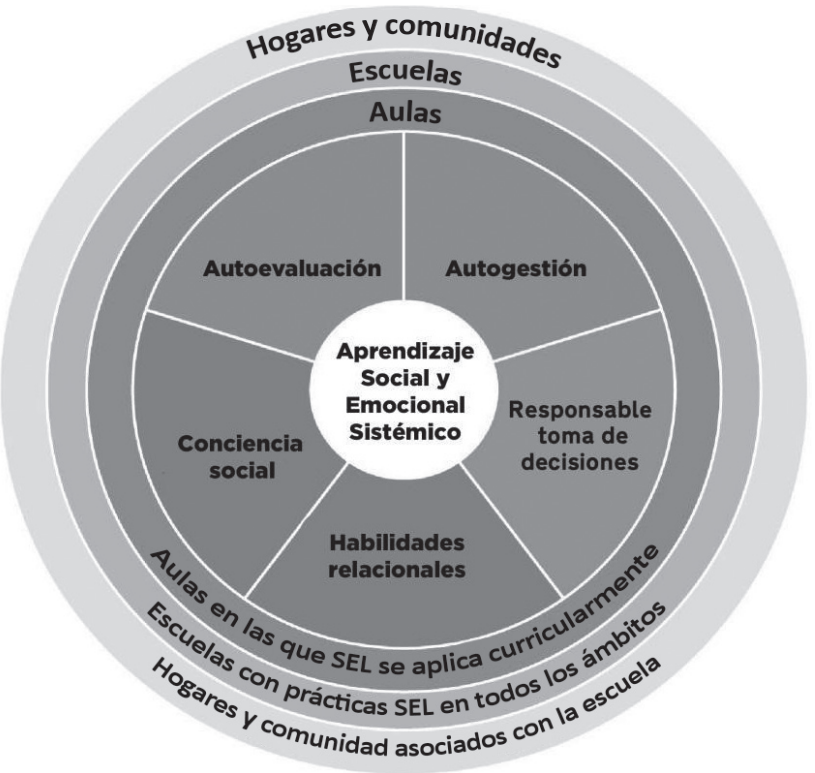

Fuente: Casel, 2017, p. 3.

\section{Las cinco grandes dimensiones de la personalidad}

La inteligencia no-cognitiva y su pariente cercana la inteligencia emocional, fundadas en las habilidades de carácter, en el cultivo de los hábitos cognitivo-comportamentales, consisten, por consiguiente, en un amplio espectro de competencias, habilidades y fortalezas que deben converger para dirigir la cognición-deliberación-decisión-acción. En nuestro caso, nos centramos en el mundo escolar y familiar (aunque tiene otras aplicaciones: profesional, laboral, comunitaria, etc.). Estas competencias, estos hábitos, deben integrarse para alcanzar resultados, pues no funcionan unas sí y las otras no. Se avanza en habilidades de carácter si se adelanta en la mayoría de cada una de ellas. El cuestionario Big Five mide estas fortalezas y detecta sus déficits en cinco factores de la personalidad, lo vemos desde distintas perspectivas y desde sus respectivas facetas (Tabla 3). Heckman y Kautz (2012) proponen un dibujo aproximado de lo que son las habilidades no-cognitivas y sus efectos. Estos investigadores han demostrado que los estudiantes, trabajadores, etc., que cuentan con estas habilidades presentan escolar y laboralmente un alto grado de desempeño no solo técnico-académico, sino también en el plano de un buen y resolutivo carácter capaz de gestionar retos y dificultades tanto materiales como relacionales, a partir de una razonable estabilidad emocional. Esta tabla ofrece una visión panorámica y global de los principales rasgos de la personalidad que están en tan estrecha relación con las habilidades de carácter, con las habilidades no-cognitivas. Presentamos un cuadro que nos ofrece una definición sintética (y limitada) de estos campos y a la vez nos ayuda a comprender su estudio, implementación y evaluación: Tabla 3. 
Tabla 3. Las dimensiones de los Cinco Grandes (Big Five) y sus facetas.

\begin{tabular}{|c|c|c|c|c|}
\hline $\begin{array}{l}\text { Los cinco gran- } \\
\text { des: factores } \\
\text { de la persona- } \\
\text { lidad }\end{array}$ & $\begin{array}{c}\text { La descripción } \\
\text { del Diccio- } \\
\text { nario de la } \\
\text { Asociación } \\
\text { Americana de } \\
\text { Psicología }^{3} \\
\end{array}$ & $\begin{array}{c}\text { Facetas (y su } \\
\text { correlativo } \\
\text { rasgo-adjetivo) }\end{array}$ & $\begin{array}{c}\text { Rasgos } \\
\text { relacionados }\end{array}$ & $\begin{array}{c}\text { Rasgos análo- } \\
\text { gos del tem- } \\
\text { peramento } \\
\text { infantil }\end{array}$ \\
\hline $\begin{array}{c}\text { Apertura a la } \\
\text { experiencia }\end{array}$ & $\begin{array}{l}\text { «La tendencia a } \\
\text { estar abierto a } \\
\text { nuevas experien- } \\
\text { cias estéticas, } \\
\text { culturales o } \\
\text { intelectuales». }\end{array}$ & $\begin{array}{l}\text { Imaginación (in- } \\
\text { tuitivo en el sen- } \\
\text { tido de creativo); } \\
\text { estética (artísti- } \\
\text { co); sensibilidad } \\
\text { (perceptivo); } \\
\text { iniciativa (am- } \\
\text { plios intereses); } \\
\text { ideas (curioso); } \\
\text { y valores (poco } \\
\text { convencional). }\end{array}$ & & $\begin{array}{l}\text { Sensibilidad } \\
\text { sensorial; placer } \\
\text { en actividades de } \\
\text { baja intensidad; } \\
\text { curiosidad. }\end{array}$ \\
\hline $\begin{array}{l}\text { Responsabili- } \\
\text { dad/tesón }\end{array}$ & $\begin{array}{l}\text { «a tendencia a } \\
\text { estar organizado, } \\
\text { a ser responsa- } \\
\text { ble y trabajador } \\
\text { constante». }\end{array}$ & $\begin{array}{l}\text { Competencia } \\
\text { (eficiente); orden } \\
\text { (organizado, pla- } \\
\text { nificado); sentido } \\
\text { del deber (me- } \\
\text { ticuloso, cum- } \\
\text { plidor); lucha } \\
\text { por los objetivos } \\
\text { (ambicioso en po- } \\
\text { sitivo); auto-dis- } \\
\text { ciplina (diligente } \\
\text { en el sentido de } \\
\text { no perezoso); } \\
\text { y deliberación } \\
\text { (prudente, re- } \\
\text { flexivo). }\end{array}$ & $\begin{array}{l}\text { Determinación; } \\
\text { perseverancia; } \\
\text { retraso de la } \\
\text { gratificación; } \\
\text { control del im- } \\
\text { pulso; coraje en } \\
\text { la tarea; persis- } \\
\text { tencia; y ética } \\
\text { del trabajo. }\end{array}$ & $\begin{array}{l}\text { Atención (falta } \\
\text { de); distractibili- } \\
\text { dad; control del } \\
\text { esfuerzo; control } \\
\text { del impulso/ } \\
\text { retraso de la } \\
\text { gratificación; } \\
\text { persistencia; y } \\
\text { actividad. }\end{array}$ \\
\hline Extroversión & $\begin{array}{l}\text { «Una orientación } \\
\text { de los propios } \\
\text { intereses y } \\
\text { energías hacia el } \\
\text { mundo exterior } \\
\text { de las personas } \\
\text { y las cosas (una } \\
\text { orientación } \\
\text { caracterizada } \\
\text { por las emocio- } \\
\text { nes positivas y } \\
\text { la sociabilidad) } \\
\text { en lugar de una } \\
\text { orientación } \\
\text { interior hacia } \\
\text { el mundo de } \\
\text { la experiencia } \\
\text { subjetiva». }\end{array}$ & $\begin{array}{l}\text { Cordialidad } \\
\text { (amistoso); socia- } \\
\text { bilidad (socia- } \\
\text { ble); asertividad } \\
\text { (seguro de sí } \\
\text { mismo); activi- } \\
\text { dad (dinámico, } \\
\text { emprendedor); } \\
\text { audacia (apasio- } \\
\text { nado); y emo- } \\
\text { ciones positivas } \\
\text { (entusiasta). }\end{array}$ & & $\begin{array}{l}\text { Vigorosidad; } \\
\text { dominancia } \\
\text { social; vitalidad } \\
\text { social; búsqueda } \\
\text { de sensaciones; } \\
\text { timidez; activi- } \\
\text { dad; emociona- } \\
\text { lidad positiva; } \\
\text { y sociabilidad/ } \\
\text { pertenencia al } \\
\text { grupo. }\end{array}$ \\
\hline
\end{tabular}




\begin{tabular}{|c|c|c|c|c|}
\hline $\begin{array}{l}\text { Disponibilidad } \\
\text { (afabilidad) }\end{array}$ & $\begin{array}{l}\text { «Una tendencia } \\
\text { a actuar de un } \\
\text { modo cooperati- } \\
\text { vo y desintere- } \\
\text { sado». }\end{array}$ & $\begin{array}{l}\text { Confianza } \\
\text { (comprensivo); } \\
\text { franqueza (des- } \\
\text { complicación); } \\
\text { altruismo (afec- } \\
\text { tuoso, genero- } \\
\text { so); obediencia } \\
\text { (no testarudo); } \\
\text { modestia (no } \\
\text { fanfarrón); y } \\
\text { disponibilidad } \\
\text { (solidario). }\end{array}$ & $\begin{array}{l}\text { Empatía; pers- } \\
\text { pectiva amplia; } \\
\text { cooperación; y } \\
\text { competitividad. }\end{array}$ & $\begin{array}{l}\text { Irritabilidad; } \\
\text { agresividad; y } \\
\text { obstinación. }\end{array}$ \\
\hline $\begin{array}{c}\text { Estabilidad/ } \\
\text { inestabilidad } \\
\text { emocional }\end{array}$ & $\begin{array}{l}\text { La estabilidad } \\
\text { emocional se } \\
\text { refleja en unas } \\
\text { «reacciones emo- } \\
\text { cionales predeci- } \\
\text { bles y coherentes } \\
\text { en ausencia de } \\
\text { rápidos cambios } \\
\text { de humor». El } \\
\text { neuroticismo «es } \\
\text { un crónico nivel } \\
\text { de inestabilidad } \\
\text { emocional y una } \\
\text { proclividad a la } \\
\text { ansiedad psicoló- } \\
\text { gica». }\end{array}$ & $\begin{array}{l}\text { Ansiedad (angus- } \\
\text { tiado); hostilidad } \\
\text { (irritable); de- } \\
\text { presión (desani- } \\
\text { mado), insegu- } \\
\text { ridad (tímido); } \\
\text { impulsividad } \\
\text { (temperamen- } \\
\text { tal); y Vulnerable } \\
\text { al estrés (frágil). }\end{array}$ & $\begin{array}{l}\text { Locus de control; } \\
\text { autoevaluación } \\
\text { cabal; autoesti- } \\
\text { ma; autoefica- } \\
\text { cia; optimismo; } \\
\text { psicopatologías y } \\
\text { trastornos Eje I } \\
\text { (DSM). }\end{array}$ & $\begin{array}{l}\text { Temor/inhibición } \\
\text { conductual (re- } \\
\text { traimiento); ti- } \\
\text { midez; irritabili- } \\
\text { dad; frustración; } \\
\text { incapacidad para } \\
\text { auto-calmarse; } \\
\text { Tristeza. }\end{array}$ \\
\hline
\end{tabular}

Fuente: Elaboración propia a partir de Heckman y Kautz, 2012; John y Srivastava, 1999.

เ

\section{Conclusión: aprender a pensar un futuro más complejo}

Recapitulemos: en el mundo de la educación parece evidente, desde hace años, que no se trata únicamente de contar con la inteligencia cognitiva (medida por el Cociente Intelectual, IQ en otros idiomas), sino que es vital también destacar con énfasis la inteligencia no-cognitiva que debe estar en su base: las habilidades de carácter. Unas habilidades de carácter que estarán luego presentes a lo largo de la vida: en secundaria, en los estudios superiores, en la vida profesional, en la vida social y también como base de una vida familiar estable.
En las investigaciones anglosajonas se destaca que las habilidades de carácter son base del capital humano (aquellas habilidades y capacidades que crean valor en el mundo profesional y se traduce en mejoras individuales, para el empleador, la empresa, la productividad de un país, etc.) (Heckman, 2011). Pero el carácter, el buen carácter, también está en la base de la vida cívica entendida como el resultado de diversos aspectos en concordancia: la consistencia de la vida familiar (que siempre tiene rendimientos sociales); la participación ciudadana; las virtudes cívicas; la ausencia de comisión de delitos; y la superación de patologías psico-sociales por parte de sus miembros (Nucci, Krettenauer y Narváez, 2014). 
Iniciamos un siglo que comienza con desafiantes retos y una creciente complejidad en diferentes ámbitos: una nueva época la de la cuarta Revolución Industrial-que exige una nueva formación para un nuevo tipo de ciudadano en un mundo cambiante. El presente siglo exige personalidades adaptativas equipadas de unas habilidades capaces de gestionar la complejidad. En este sentido, muchos expertos hablan de las habilidades del siglo XXI-21st Century skills (National Research Council, 2012) con la voluntad de enfatizar planos de la inteligencia operativa tan centrales como las habilidades de pensamiento crítico, la toma de decisiones y la resolución de problemas en la línea de las funciones ejecutivas. Son unas habilidades que ponen de relieve el enfoque competencial y que son demandadas por organismos internacionales como la OCDE (Ananiadou y Claro, 2009). Ya no se trata solo de cultivar habilidades estratégicas sino también de equipar a los nuevos ciudadanos con actitudes interiores resolutivas, mentalidades ágiles y flexibles visiones del mundo.

Imbricadas, pues, con las habilidades de carácter están las actitudes, las creencias: aquello que en el mundo anglosajón se denomina mindset. Estamos en el plano de las convicciones interiores, los estilos atributivos y el tono psicológico, que: 0 bien pueden alentar el trabajo y la motivación intrínseca; o bien pueden desanimar y paralizar. En esta esfera se mueve la teoría de la autodeterminación de Deci y Ryan (2000) que tan bien ha estudiado la motivación intrínseca. Es también el plano de la auto-eficacia (self-efficacy) investigada por Bandura (1997). Los menores, los estudiantes y los futuros ciudadanos deben incorporar unas visiones de sí mismos y del mundo que les permita ganar en competencia, autonomía y capacidad de relación sin dejarse intoxicar por un entorno a veces alienante y destructivo.

Algunos contenidos de los medios digitales pueden actuar negativamente $\mathrm{y}$ además ejercer un papel distractor y ansiógeno (Hoge, Bickham y Cantor, 2017) a través de distintos dispositivos, donde destaca el excesivo uso del móvil y de las redes sociales. Las distracciones, la carencia de atención, la desconcentración en el aula y en el estudio son dificultades que hoy afronta la educación cada vez de un modo más agudo (Scherer y Hatlevik, 2017).

Sin embargo, las perspectivas de cambio son alentadoras. Estamos en un campo educable. Un buen ejemplo son los estudios sobre la perseverancia apasionada, grit, realizados por Duckworth (2013). Es un tema amplio con diferentes ramificaciones que aquí no podemos consignar. También se podría incluir el campo de la necesidad de silencio, de la capacidad de auto-calmarse, de la habilidad de entrar en el interior de uno mismo para concentrarse, alcanzar la atención plena y canalizar el estrés. Nuestros estudiantes, desde bien pequeños, pueden estar muy estresados y hay que alcanzar el descanso estabilizador que favorece el aprendizaje como ha estudiado Kabat-Zinn (2003) en la amplia esfera del mindfulness. El carácter, la actitud, la resiliencia, la paz interior son las condiciones que posibilitan el mejor rendimiento escolar. En estas últimas líneas estamos hablando de la estabilidad psíquica como 
una de las bases del aprendizaje, que se concreta en la superación de la ansiedad, el miedo, la tristeza, el bajo auto-concepto 0 el cambio continuado de tono emocional. Parte de este campo puede ser medido, de nuevo, por el Big Five Questionnaire (BFQ) en su factor de personalidad estabilidad/inestabilidad emocional (neuroticism/emotional stability).

El objetivo central de este trabajo es poner en la agenda educativa la importancia de esta inteligencia del carácter. Es preciso invitar a la discusión sobre estos temas a los agentes educativos. Pensamos que esta inteligencia del carácter está un poco olvidada: no es solo una formación moral sino también intelectual. Y el primer paso para promover las habilidades de carácter es certificar que realmente influyen en el éxito escolar y el bienestar emocional. El cuerpo de investigación sobre este tema es escaso pero prometedor (Khine y Areepattamannil, 2016; Roberts et al., 2015). Concluyamos: existen diversos instrumentos de medición de este campo - Big Three, MPQ, Big Nine-- pero en estas líneas hemos propuesto uno de los más completos y testados: el Big Five Questionnaire en su versión para el ámbito escolar y para el mundo hispano, es decir, el BFQ-N más arriba citado. Y a su vez contamos con el instrumento BRIEF 2, también validado para el mundo hispano e infanto-juvenil. BRIEF 2 se dirige a evaluar las FE (funciones ejecutivas) que consideramos que están en el núcleo de las habilidades de carácter. Una triangulación que abre la puerta a la voz de las familias pues el BRIEF 2 cuenta con una versión para profesores y otra para padres.

\section{Notas}

${ }^{1}$ «Throughout this paper we use the term character skills to describe the personal attributes not thought to be measured by IQ tests or achievement tests. These attributes go by many names in the literature, including soft skills, personality traits, non-cogniti. ve skills, non-cognitive abilities, character, and so. cio-emotional skills. These different names conno. te different properties» (versión original en inglés) (Heckman y Kautz, 2013, p. 3).

${ }^{2}$ «Non-cognitive is, of course, a misnomer. Every psychological process is cognitive in the sense of relying on the processing of information of some kind» (versión original en inglés) (West et al., 2016, p. 149).

${ }^{3}$ Estas definiciones proceden del Diccionario de la Asociación Americana De Psicología (VandenBos, 2015).

\section{Referencias bibliográficas}

Ainsworth, M. D. y Marvin, R. S. (1995). On the shaping of attachment theory and research: An interview with Mary DS Ainsworth (Fall 1994). Monographs of the society for research in child development, 60 (2-3), 3-21. doi: http://dx.doi. org $/ 10.2307 / 1166167$

Alcover, C. y Rodríguez, F. (2012). Plasticidad Cerebral y Hábito en William James: un Antecedente para la Neurociencia Social. Psychologia Latina, 3 (1), 1-9.

Althof, W. y Berkowitz, M. W. (2006). Moral education and character education: Their relationship and roles in citizenship education. Journal of moral education, 35 (4), 495-518. doi: https:// doi.org/10.1080/03057240601012204

Álvarez-Hevia, D. M. (2018). Aproximación crítica a la Inteligencia Emocional como discurso dominante en el ámbito educativo. revista española de pedagogía, 76 (269), 7-23. doi: https://doi.org/10.22550/REP76-1-2018-01

Ananiadou, K. y Claro, M. (2009). 21st Century Skills and Competences for New Millennium Learners in OECD Countries. OECD Education Working Papers, 41. doi: https://doi. org/10.1787/19939019

Bandura, A. (1977). Self-efficacy: toward a unifying theory of behavioral change. Psychological review, 84 (2), 191-215. doi: https://doi. org/10.1016/0146-6402(78)90002-4 
Bernacer, J. y Murillo, J. I. (2014). The Aristotelian conception of habit and its contribution to human neuroscience. Frontiers in human neuroscience, 8, 1-10. doi: https://doi.org/10.3389/ fnhum.2014.00883

Bernstein, B. (1989). Clases, códigos y control (Vol. 1). Madrid: Ediciones Akal.

Bourdieu, P. (1986). Habitus, code et codification. Actes de la recherche en sciences sociales, 64 (1), 40-44. doi: https://doi.org/10.3406/ arss.1986.2335

Bourdieu, P. (1979). La distinction. Critique sociale du jugement. Paris: Minuit.

Bowlby, J. (1969). Attachment and loss: Vol. 1. Attachment. New York: Basic Books.

Bowlby, J. (1973). Attachment and loss: Vol. 2. Separation: Anxiety and anger. New York: Basic Books.

Bronfenbrenner, U. (2009). The ecology of human development. Cambridge: Harvard University Press.

CASEL (2017). Educating Hearts, Inspiring Minds. Core SEL competencies. Recuperado de https://casel.org/core-competencies/ (Consultado el 15/01/2018).

Carrasco-Ortiz, M. Á., Holgado-Tello, F. P. y Del Barrio-Gandara, M. V. (2005). Dimensionalidad del cuestionario de los cinco grandes (BFQ-N) en población infantil española. Psicothema, 17 (2), 286-291.

Center on the Developing Child at Harvard University (2017). Three Principles to Improve Outcomes for Children and Families. Recuperado de https://bit.ly/2gSMqh2 (Consultado el 20/02/2018).

Chen, Q. y Yan, Z. (2016). Does multitasking with mobile phones affect learning? A review. Computers in Human Behavior, 54, 34-42. doi: https://doi.org/10.1016/j.chb.2015.07.047

Damasio, A. R. (1996). El error de Descartes. Barcelona: Crítica.

De Bofarull, I. (2005). Ocio y tiempo libre: un reto para la familia. Pamplona: Eunsa.

De Bofarull, I. y Coma, M. A. (2017). Possibilities and limitations of digital multitasking for school homework. Quaderns de Polítiques Familiars, 3, 48-58.
Diamond, A. (2013). Executive functions. Annual review of psychology, 64, 135-168. doi: https:// doi.org/10.1146/annurev-psych-113011-143750

Diamond, A. (2014). Want to optimize executive functions and academic outcomes? Simple, just nourish the human spirit. Minnesota Symposia on Child Psychology, 37, 205-232.

Diamond, A. y Ay Lee, K. (2011). Interventions shown to aid executive function development in children 4 to 12 years old. Science, 333 (6045), 959-964. doi: https://doi.org/10.1126/ science. 1204529

Duckworth, A. L. (2013). True grit. The Observer, $26(4), 1-3$.

Durlak, J. A., Dymnicki, A. B., Taylor, R., Weissberg, R. P. y Schellinger, K. B. (2011). The impact of enhancing students' social and emotional learning: A meta-analysis of school-based universal interventions. Child Development, $82,405-432$.

Dweck, C. S. (2006). Mindset: The new psychology of success. New York: Random House Incorporated.

Elias, M. J., Leverett, L., Duffell, J. C., Humphrey, N., Stepney, C. y Ferrito, J. (2015). Integrating SEL with related prevention and youth development approaches. En J. A. Durlak, C. E. Domitrovich, R. P. Weissberg y T. P. Gullotta (Eds.), Handbook for social and emotional learning: Research and practice (pp. 33-49). New York: Guilford.

Galán-Rodríguez, A. (2010). El apego: Más allá de un concepto inspirador. Revista de la Asociación Española de Neuropsiquiatría, 30 (4), 581-595.

Gervilla, E. (2000). Un modelo axiológico de educación integral. revista española de pedagogía, 58 (215), 39-57.

Gioia, G. A., Isquith, P. K., Guy, S. C. y Kenworthy, L. (2000). Test review behavior rating inventory of executive function. Child Neuropsychology, 6 (3), 235-238.

Hanscombe, K. B., Haworth, C. M., Davis, O. S., Jaffee, S. R. y Plomin, R. (2011). Chaotic homes and school achievement: a twin study. Journal of Child Psychology and Psychiatry, and Allied Disciplines, 52 (11), 1212-1220. doi: https://doi. org/10.1111/j.1469-7610.2011.02421.x 
Heckman, J. J. (2011). The Economics of Inequality: The Value of Early Childhood Education. American Educator, 35 (1), 31-35.

Heckman, J. J. y Kautz, T. (2012). Hard evidence on soft skills. Labour economics, 19 (4), 451-464.

Heckman, J. y Kautz, T. (2013). Fostering and Measuring Skills: Interventions That Improve Character and Cognition. National Bureau of Economic Research, 19656. doi: https://doi. org/10.3386/w19656

Hoge, E., Bickham, D. y Cantor, J. (2017). Digital media, anxiety, and depression in children. Pediatrics, 140 (2), S76-S80. doi: https://doi. org/10.1542/peds.2016-1758G

James, W. (1899). Talks to Teachers on Psychology and to Students on Some of Life's Ideals. Boston: Harvard University Press.

Jennings, P. A. y Greenberg, M. T. (2009). The prosocial classroom: Teacher social and emotional competence in relation to student and classroom outcomes. Review of Educational Research, 79 (1), 491-525.

John, O. P. y Srivastava, S. (1999). The Big Five trait taxonomy: History, measurement, and theoretical perspectives. En L. A. Pervin y O. P. John (Eds.), Handbook of personality: Theory and research (pp. 102-138). New York: Guilford Press.

Kabat-Zinn, J. (2003). Mindfulness-based interventions in context: past, present, and future. Clinical psychology. Science and practice, 10 (2), 144-156.

Kautz, T., Heckman, J. J., Diris, R., Ter Weel, B. y Borghans, L. (2014). Fostering and measuring skills: Improving cognitive and non-cognitive skills to promote lifetime success. National Bureau of Economic Research, 20749, 1-118. doi: https://doi.org/10.3386/w20749

Khine, M. S. y Areepattamannil, S. (Eds.) (2016). Non-cognitive Skills and Factors in Educational Attainment. Rotterdam: Sense Publishers.

Lickona, T. (1991). Educating for character: How our schools can teach respect and responsibility. New York: Bantam Books.

Maldonado-Belmonte, M. J. (2016). Adaptación del BRIEF (Behavior Rating Inventory of Executive Function) a población española y su utilidad para el diagnóstico del trastorno por déficit de atención-hiperactividad subtipos inatento y combinado (Tesis doctoral). Universidad Complutense de Madrid).

Malikail, J. (2003). Moral character: hexis, habitus and 'habit'. An Internet Journal of Philosophy, 7, 1-22.

McEwen, B. S. (2016). In pursuit of resilience: stress, epigenetics, and brain plasticity. Annals of the New York Academy of Sciences, 1373 (1), 56-64.

Moullin, S., Waldfogel, J. y Washbrook, E. (2014). Baby Bonds: Parenting, attachment and a secure base for children. The Sutton Trust. Recuperado de http://dera.ioe.ac.uk/30276/1/babybonds-final-1.pdf (Consultado el 24/10/2018).

National Academies of Sciences, Engineering, and Medicine (2016). Parenting matters: Supporting parents of children ages 0-8. Washington, D.C.: The National Academies Press.

National Research Council (2012). Education for life and work: Developing transferable knowledge and skills for the 21st century. Washington, D.C.: The National Academies Press.

Nucci, L., Krettenauer, T. y Narváez, D. (Eds.) (2014). Handbook of moral and character education. New York: Routledge.

OECD (2013). PISA 2012 Results: Ready to Learn (Volume III): Students' Engagement, Drive and Self-Beliefs. Paris: OECD Publishing.

Palmero-Cámara, C., Jiménez-Martín, J. y Jiménez-Eguizábal, A. (2015). Ocio, Política y Educación. Reflexiones y retos veinticinco siglos después de Aristóteles. revista española de pedagogía, 73 (216), 5-21.

Perry, B. D. (2009). Examining child maltreatment through a neurodevelopmental lens: Clinical applications of the neurosequential model of therapeutics. Journal of Loss and Trauma, 14 (4), 240-255.

Real Academia Española (2017). Diccionario de la lengua española (23.1 ed.). Recuperado de http://www.rae.es/ (Consultado el 25/10/18).

Roberts, R. D., Martin, J. y Olaru, G. (2015). A Rosetta Stone for noncognitive skills: Understanding, assessing, and enhancing noncognitive skills in primary and secondary education. New York: Asia Society and ProExam. 
Ryan, R. M. y Deci, E. L. (2000). Intrinsic and extrinsic motivations: Classic definitions and new directions. Contemporary educational psychology, 25 (1), 54-67.

Scherer, R. y Hatlevik, O. E. (2017). «Sore eyes and distracted» or «excited and confident»?-The role of perceived negative consequences of using ICT for perceived usefulness and self-efficacy. Computers \& Education, 115, 188-200. doi: https://doi.org/10.1016/j.compedu.2017.08.003

Shaheen, S. (2014). How child's play impacts executive function-related behaviors. Applied Neuropsychology: Child, 3 (3), 182-187. doi: https:// doi.org/10.1080/21622965.2013.839612

Shonkoff, J. P., Boyce, W. T. y McEwen, B. S. (2009). Neuroscience, molecular biology, and the childhood roots of health disparities: building a new framework for health promotion and disease prevention. Jama, 301 (21), 2252-2259. doi: https://doi.org/10.1001/jama.2009.754

Soprano, A. M. (2003). Evaluación de las funciones ejecutivas en el niño. Revista de Neurología, 37 (1), 44-50.

Taylor, R. D., Oberle, E., Durlak, J. A. y Weissberg, R. P. (2017). Promoting Positive Youth Development Through School-Based Social and Emotional Learning Interventions: A Meta-Analysis of Follow-Up Effects. Child development, 88 (4), 11561171. doi: https://doi.org/10.1111/cdev.12864

Thoilliez, B. (2013). El pensamiento pedagógico de William James: el tactful teacher y la educación moral. Foro de Educación, 11 (15), 83-102. doi: http://dx.doi.org/10.14516/ fde.2013.011.015.004

Tough, P. (2014). Cómo triunfan los niños: Determinación, curiosidad y el poder del carácter. Madrid: Palabra.

Tough, P. (2016). Helping children succeed: What works and why. New York: Random House.
VandenBos, G. R. (Ed.) (2015). American Psychological Association. APA Dictionary of Psychology. Washington: American Psychological Association.

Vernon-Feagans, L., Willoughby, M. y Garrett-Peters, P. (2016). Predictors of behavioral regulation in kindergarten: Household chaos, parenting, and early executive functions. Developmental psychology, 52 (3), 430-441.

Vigo, A. G. (2012). Deliberación y decisión según Aristóteles. Tópicos, 43, 51-92.

West, M. R., Kraft, M. A., Finn, A. S., Martin, R. E., Duckworth, A. L., Gabrieli, C. F. y Gabrieli, J. D. (2016). Promise and paradox: Measuring students' non-cognitive skills and the impact of schooling. Educational Evaluation and Policy Analysis, 38 (1), 148-170. doi: https://doi. org/10.3102/0162373715597298

\section{Biografía del autor}

Ignasi de Bofarull es Licenciado en Geografía e Historia por la Universitat Autónoma de Barcelona y Doctor en Humanidades por la Universitat Internacional de Catalunya. Actualmente ejerce como Profesor Contratado Doctor de Sociología de la Familia y de la Educación en la Facultad de Educación de la UIC, Investigador del Instituto del Estudios Superiores de la Familia de la UIC y Director del grupo de investigación de la Generalitat de Catalunya denominado Parentalitat, Igualtat i Conciliació.

iD https://orcid.org/0000-0003-4896-1997 


\section{revista española de pedagogía año 77, $n^{\circ}$ 272, enero-abril 2019}

Spanish Journal of Pedagogy

year 77, n. 272, January-April 2019

Sumario*

Table of Contents **

\section{Estudios \\ Studies}

Francisco López Rupérez, Isabel Garcia García,

\section{Eva Expósito Casas}

Rendimiento en ciencias, concepciones epistémicas y vocaciones STEM en las comunidades autónomas españolas. Evidencias desde PISA 2015, políticas y prácticas de mejora

Performance in Sciences, epistemic conceptions and STEM vocations in the Spanish Autonomous Communities. Evidence from PISA 2015, improvement policies and practices

\section{Zaida Espinosa Zárate}

El cultivo de la creatividad para el diálogo intercultural

Cultivating creativity for intercultural dialogue

Ignasi de Bofarull

Carácter y hábitos para el aprendizaje:

definición y proyecto de medición

Character and learning habits:

definition and measurement proposal

\section{9}

David Luque

Desarrollos interpretativos de la filosofía

de la educación en la tradición anglófona:

un intento de sistematización

Interpretive developments of the philosophy

of education in the anglophone tradition:

an attempt to systematise them

\section{Notas \\ Notes}

\section{María Moralo, Manuel Montanero}

Aprendizaje con y sin error en estudiantes con TEA

9 Learning with and without errors in students with ASD

85

\section{Antonio Portela Pruaño,}

José Miguel Nieto Cano, Ana Torres Soto

La reincorporación formativa de jóvenes

que abandonan tempranamente la educación:

47 relevancia de su trayectoria previa

Re-engagement in education and training

of young people who leave education early:

the importance of earlier prior trajectories

\footnotetext{
* Todos los artículos están también publicados en inglés en la página web de la revista: https://revistadepedagogia.org.

** All the articles are also published in English on the web page of the journal: https://revistadepedagogia.org.
} 
Oihane Fernández-Lasarte, Eider Goñi, Igor Camino, Estibaliz Ramos-Díaz

Apoyo social percibido e implicación escolar del alumnado de educación secundaria

Perceived social support and school engagement in secondary students

Jesús-Miguel Muñoz-Cantero, Luisa Losada-Puente

Validación del constructo de autodeterminación a través de la escala ARC.INICO para adolescentes Validation of the construct of self-determination through the ARC.INICO scale for teenagers

Ma Inmaculada López-Núnez, Susana Rubio-Valdehita, Eva Má Diaz-Ramiro

Cambios producidos por la adaptación al EEES en la carga de trabajo de estudiantes de Psicología Changes in Psychology students' workload due to alignment with the EHEA

\section{Reseñas bibliográficas}

Naval, C. y Arbués, E. (Eds.). Hacer la Universidad en el espacio social (Emanuele Balduzzi). Mínguez, R. y Romero, E. (Coords.). La educación ciudadana en un mundo en transformación: miradas y propuestas (Marina Pedreño Plana). Bellamy, F.-X. Los desheredados: por qué es urgente transmitir la cultura (Esteban López-Escobar). Pallarés, M., Chiva, 0., López Martín, R. y Cabero, I. La escuela que llega. Tendencias y nuevos enfoques metodológicos (Juan Carlos San Pedro Velado).

\section{Informaciones}

El Profesor Giuseppe Mari. In memoriam (Emanuele Balduzzi); III Conferencia Internacional de EuroSoTL: "Explorando nuevos campos a través de un enfoque académico de la enseñanza y el aprendizaje";

123 Una visita a la hemeroteca (Ana González-Benito); Una visita a la red (David Reyero).

\section{Instrucciones para los autores}

Instructions for authors 201 


\section{Character and learning habits: definition and measurement proposal \\ Carácter y hábitos para el aprendizaje: definición y proyecto de medición}

Ignasi de BOFARULL, PhD. Assistant Professor. Universitat Internacional de Catalunya (ibofarul|@uic.es).

\begin{abstract}
:
This work examines how students' character, habits, and mindset influence teaching-learning processes. Until recently character education was a matter of moral and civic education, but recent research into non-cognitive skills and social-emotional learning reflects how these personality traits give steadiness to school teaching-learning processes. Neuroscience here emphasises the value of executive functions: attention, inhibitory control, and planning are moments where the student unfolds her learning. Classical intelligence (IQ) focuses on analytical understanding, a specific moment; character intelligence focuses on the volitional processes that create the intellectual work that begins in the classroom and ends with planning of study at home. The first objective is to define non-cognitive skills, executive functions, and character, related frameworks that are present in school and family life. After this, the second objective is to assess how the social-family environment affects
\end{abstract}

these processes. The third objective, in parallel with carrying out the study, is to propose tools to measure these strengths in elementary school: BFQ-N and BRIEF 2. If character education models for learning are proposed, tools should be offered to measure it intended to test the success of the programmes. The conclusion identifies a major initiative: universities, schools, and educational agents should think about a new integrated model of education for their students based on this convergence between character and classical intelligence. School failure and dropout have academic explanations but also family and personal ones. This complex and changing third millennium requires robust and flexible skills to face the challenges of a society that has still not shown us where it is going.

Keywords: character, habits, non-cognitive skills, executive functions, social and emotional learning, attachment, mind-set, whole education, 21st century skills.

Revision accepted: 2018-03-30.

This is the English version of an article originally printed in Spanish in issue 272 of the revista española de pedagogía. For this reason, the abbreviation EV has been added to the page numbers. Please, cite this article as follows: De Bofarull, I. (2019). Carácter y hábitos para el aprendizaje: definición y proyecto de medición | Character and learning habits: definition and measurement proposal. Revista Española de Pedagogía, 77 (272), 47-65. doi: https://doi.org/10.22550/REP77-1-2019-03 


\section{Resumen:}

Este trabajo estudia cómo influye el carácter, los hábitos y la actitud del estudiante en los procesos de enseñanza-aprendizaje. Hasta hace poco la educación del carácter constituía un asunto de educación moral y cívica. Ahora, las nuevas investigaciones sobre las habilidades no-cognitivas y el aprendizaje socioemocional reflejan cómo estos planos de la personalidad dan consistencia a los procesos de enseñanza-aprendizaje escolares. Las neurociencias subrayan aquí el valor de las funciones ejecutivas: la atención, la tenacidad y la planificación son momentos donde el estudiante despliega su aprendizaje. La inteligencia clásica (coeficiente intelectual, CI) pone el acento en la comprensión analítica, un momento puntual; la inteligencia del carácter pone el foco en los procesos volitivos que fraguan el trabajo intelectual que empieza en el aula y acaba en la planificación del estudio en el hogar. Una vez definidas las habilidades no-cognitivas, las funciones ejecutivas y el carácter, marcos emparentados entre sí y presentes en la vida escolar y familiar, el segundo objetivo ha sido valorar cómo el ambiente socio-familiar interviene agudamente en estos procesos. El tercer objetivo ha sido, siempre en paralelo con el despliegue del estudio, proponer las herramientas que midan estas fortalezas en la educación primaria: BFQ-N y BRIEF 2. Si se proponen modelos de formación del carácter para el aprendizaje se deben ofrecer herramientas para su medición orientadas a testar el éxito de estos programas. La conclusión apunta a una iniciativa de calado: la universidad, la escuela y los agentes educativos deben pensar un nuevo modelo de educación integral de sus estudiantes desde esta convergencia entre carácter e inteligencia clásica. El fracaso y el abandono escolar tienen razones académicas, pero también familiares y personales. Este tercer milenio, complejo, cambiante, necesita habilidades robustas y flexibles para hacer frente a los retos de una sociedad que no nos desvela aún a dónde va.

Descriptores: carácter, hábitos, habilidades no-cognitivas, funciones ejecutivas, aprendizaje socio-emocional, apego, actitud, educación integral, habilidades para el siglo XXI.

\section{Character skills}

When a child is born, it has a biologically-shaped temperament that will be moulded over time into a character which is the product of habits acquired and shaped by its family, school, and social environment. The purpose of school is, in our opinion, for students to develop a character that is the sum of habits acquired in repeated actions that are cognitive, ethical, and behavioural in nature. We frame this objective in the later years of early-childhood education and in all of primary education. We refer to learning habits that should take root at the cerebral and neuronal levels. As James realised as long ago as the late nineteenth century (James, 1899; Alcover \& Rodríguez, 2012), habits form new and more agile and supple learning-behaviour 
thanks to neuroplasticity, a modification of the neuronal networks and of the physical structure of the brain. The result is that the student will establish a second nature, a new cognitive and behavioural functioning that can fix thinking skills of the highest level which will in turn be able to continue learning.

These concepts of habit and character are anchored in a Western tradition that began with Aristotle's philosophy, in particular the Nicomachean Ethics (Malikail, 2003; Bernacer \& Murillo, 2014), and which is still of great importance today. Training habits as the basis of education is a thread running from Aristotle to neurosciences, passing through James's insights. Good character, in short, is built on the habits and strengths that enable us to carry ourselves correctly through life, achieving our family, career, and civic goals. Furthermore, a good education in citizenship should not ignore the fact that committed, participatory, and critical citizens who can build a quality democracy must be educated to have a good character (Althof \& Berkowitz, 2006). Citizenship without civic virtues, in Aristotelian terms, can endanger the sustainability of a model of society. We are, therefore, speaking of integrated education (Gervilla, 2000).

Personality psychology has been describing these processes for decades. We intend to define and propose a means of measuring how character, habits, and personal strengths affect the learning process at school. We say learning process as learning at school is sequential and it is not just in class that it develops (or deteriorates) but also in family and social settings, while studying, and in the planning of tasks. At present it has to compete with distracting digital activity such as social media (Chen \& Yan, 2016).

What is currently happening in school life is that people often forget how character influences learning. The Real Academia Española dictionary defines character as «strength and nobility of spirit natural to someone, resolve, energy.» Character is a personal motor that constantly regulates the cognition-deliberation-decision-action sequence (Vigo, 2012). And the moment of deliberation-decision is not just a rational step but is also a choice loaded with the emotions, beliefs, and attitudes in play (Damasio, 1996). In brief (and returning to the pedagogical aspect of James), schools must train students in the skill of knowing-deliberating-deciding-acting and in so doing must take into account the most intelligent emotions, beliefs, and attitudes based on rooted and wise habits that tactful teachers can awaken (Thoilliez, 2013). In the USA, when discussing character education, the head, heart, hands trinity is cited (Lickona, 1991). The best thing for teachers and students is to be motivated to study a discipline or task in depth. Doing this in an abstract way and merely through a sense of duty is less appealing. Although a sense of duty can sometimes support passion and motivation, as the final objective is deeply exciting. Every day teachers confirm that students' personal strengths, positive working habits, intrinsic motivations, and self-regulation are positive- 
ly reflected in their marks. And indifference, inattention, and impulsiveness, in contrast, lead to academic failure. At a more technical level, it is known that these character skills, acting in different personality areas are, when they converge, partly conditions that make better learning possible:

Throughout this paper we use the term character skills to describe the personal attributes not thought to be measured by IQ tests or achievement tests. These attributes go by many names in the literature, including soft skills, personality traits, non-cognitive skills, non-cognitive abilities, character, and socio-emotional skills. These different names connote different properties (Heckman \& Kautz, 2013, p. 3).

\section{Self-regulation and executive functions}

We now return to the relationship between habit, learning, and neuroplasticity. The sciences of neurodevelopment place great emphasis on this: self-regulation is a vital part of learning and as time passes is also part of a balanced and emotionally stable life (Shonkoff, Boyce, \& McEwen, 2009). Self-regulation, also known as inhibitory control, is a basic ability to be able to cope in almost all areas of life. At the same time it is one of the foundations of the executive functions (EF from now on) that form the basis of character skills (Diamond, 2013; Diamond \& Lee, 2011). EFs depend for their functioning on the prefrontal cortex, the initial and substantial development of which occurs in the first three months of life (McEwen, 2016).
EFs (working memory, inhibitory control, and flexible thinking-behaviour) could be defined as a set of higher-order cognitive-behavioural competences that reason and analyse the different pieces of content at school (and in almost all life contexts). These decisions are often then turned into tasks that must be carried out in short-, medium- and long-term plans. EFs also continue to operate there: in planning and achieving objectives at school, in the family, and in life (Diamond, 2014). To measure character and learning skills in general we propose a tool that evaluates personality overall in these school ages: BFQ-N (part 6). This will be considered below. Nonetheless, there is a questionnaire that specifically measures $\mathrm{EF}$ with great precision. We propose a research project to evaluate character skills which, on those lines, seeks triangulation from different perspectives and consequently uses converging instruments. Therefore, we suggest using BRIEF 2 to specifically measure EFs (Gioia, Isquith, Guy, \& Kenworthy, 2000).

BRIEF (behaviour rating inventory of executive function) is a scale comprising two questionnaires, one for parents and another for teachers, designed to evaluate EFs in the home and the school respectively (Soprano, 2003, p. 45). See Table 1.

The Spanish version of BRIEF 2 gives the school's perspective, together with the family's, between the ages of 5 and 18. We should recall that in this piece, as stated above in the opening lines, we aim to evaluate EFs at the end of early years education and above all in primary education (Maldonado-Belmonte, 2016). 
TABLE 1. Areas of the executive functions explored by the BRIEF scale.

\begin{tabular}{|c|l|}
\hline Inhibition & $\begin{array}{l}\text { Ability to control impulses and stop engaging in behaviour at the } \\
\text { appropriate moment. }\end{array}$ \\
\hline Shift & $\begin{array}{l}\text { Ability to make transitions and tolerate changes, flexibility to solve problems } \\
\text { and move focus of attention from one topic to another when needed. }\end{array}$ \\
\hline control & The influence of EFs on the expression and control of emotions. \\
\hline Initiative & $\begin{array}{l}\text { The ability to initiate a task or activity without being made to do so. This } \\
\text { includes aspects such as the ability to generate problem-solving ideas, } \\
\text { responses, or strategies independently. }\end{array}$ \\
\hline Working & $\begin{array}{l}\text { The ability to hold information in mind with the aim of completing a } \\
\text { task, recording and storing information, and creating objectives. Working } \\
\text { memory is essential for carrying out multiple or simultaneous activities, } \\
\text { such as arithmetic calculations or following complex instructions. }\end{array}$ \\
\hline $\begin{array}{c}\text { Organization } \\
\text { and planning }\end{array}$ & $\begin{array}{l}\text { These are important for problem solving. Organization involves the ability } \\
\text { to order information and identify the main ideas or the key concepts in } \\
\text { learning tasks or when trying to communicate information, either orally or } \\
\text { in writing. Planning involves setting an objective and determining the best } \\
\text { route to achieve it, often through a series of appropriately ordered steps. }\end{array}$ \\
\hline Organization \\
of materials & $\begin{array}{l}\text { Another aspect of organization is the ability to order items in one's } \\
\text { environment and also to maintain order in work elements, toys, cupboards, } \\
\text { desks, and other places where things are kept, as well as being sure that the } \\
\text { materials needed to do a task are actually available. }\end{array}$ \\
\hline Monitoring & $\begin{array}{l}\text { This has two aspects: } \\
\text { a. The first refers to the habit of monitoring one's own performance while } \\
\text { carrying out a task or immediately after completing it in order to make } \\
\text { sure the proposed target has been attained adequately. } \\
\text { b. The second aspect, which the authors call self-monitoring, reflects the } \\
\text { child's awareness of how her behaviour affects others. }\end{array}$ \\
\hline
\end{tabular}

Source: Adapted from Soprano, 2003, p. 45.

\section{Cognitive and non-cognitive skills and measuring them}

Having reviewed the literature on character skills, we will now consider how they are measured. It is widely known that learning at school is not restricted to a pre-set curriculum; it does not just involve the analytic and cognitive incorporation of competences, subjects, and objectives by the students. The function of school is not just to obtain the stark qualifications every student gets and which are summarised in the evaluation by their teachers who certify their command of the curric- ulum content. Learning and intelligence, understood in the classical sense, are measured by the countless existing tests that establish what we know as the intelligence quotient (IQ). But this strictly academic cognitive intelligence is not all there is. There are also non-cognitive skills personality, character - that support and underpin academic intelligence, forming a new more comprehensive intelligence. To discuss non-cognitive skills as the basis of academic intelligence seems to be a contradiction. The personality and character features that have an effect on non-cognitive 
skills are also cognition although this is in its most volitional and behavioural aspect. However, this name captures everything that is not included in what is strictly referred to as cognitive skills: «non-cognitive is, of course, a misnomer. Every psychological process is cognitive in the sense of relying on the processing of information of some kind» (West et al., 2016, p. 149).

In English these skills are also referred to as soft-skills because of their malleability. These skills can, therefore, be shaped; they are not fixed. They are teachable skills that can be cultivated and measured using various instruments. Therefore, we are facing two types of skills - they could also be called intelligences - that are at the base of learning. The first type (IQ) is more cognitive, analytic, and comprehensive to put it simply. The second type, however, goes beyond what has always been understood as cognitive parcels and they promote good learning outcomes by improving habits: habits and attitudes such as perseverance, concentration, and focus on study as well as positive beliefs in one's own ability that have a significant motivational impact. This is not a new not-just-cognitive type of intelligence that has only been theorised but instead it is a new way of understanding intelligence that can be observed and can be measured in terms of personality and character traits.

For some time now it has been possible to quantify these features from the age of 17 , and one of the most important instruments -among others - for doing so is the big five personality questionnaire (BFQ) (John \& Srivastava, 1999). In any case, our interest is learning and the study of character intelligence and social-emotional intelligence at the end of early years schooling and in primary school in particular (5-12 years). To this end, there is a Spanish version of $B F Q$ for students aged from 8 to 17. This is the BFQ-N, a questionnaire that presents the psychometric requirements when the informants are the children themselves (Carrasco-Ortiz, Holgado-Tello, \& Del Barrio-Gandara, 2005). In the sixth section, the factors and aspects measured by the BFQ are considered in greater depth.

\section{Socio-family environment, educational achievement, and achievement gap}

These teaching-learning processes at school are often based on skills and personality features that predate school and continue in parallel during school years. They are rooted in the family and social environment (Bronfenbrenner, 2009). An environment where the role of the parents and closest carers stands out (Moullin, Waldfogel, \& Washbrook, 2014). If children's family environments are unstable, stressful, and chaotic, these character skills might be harmed (Perry, 2009), for example, in the areas of impulsiveness and attention difficulties. In contrast, the homes that are best organised, affectionate, and most predictable facilitate the future students' learning from the first months of life. Thanks to their order, predictability, cohesiveness, and healthy habits, these families facilitate the acquisition of routines and skills along with high academic performance 
(Hanscombe, Haworth, Davis, Jaffee, \& Plomin, 2011). Nonetheless, these studies should not make us think that high incomes are the primary variable that facilitates the emergence of character skills. It may be the case that the interactions, order, and consistence in the home are found in the attitudes of families with an economic level that is not high but instead is medium or medium-low (Tough, 2014 and 2016). At the same time, there may also be high-income homes that are so chaotic that learning becomes difficult among other reasons because the cultivation of the executive functions has deteriorated (Vernon-Feagans et al. 2016). The family environment and learning in pre-school and school years is shaped by various psychological perspectives and conceptual frameworks. We will consider the ones we regard as most relevant. One clear example of this social-family root that could shape learning is the attachment link which is located in the parental nucleus (in parent-child interactions) and is shaped in the first two years of life. In any case, its subtle and profound reality continues to be present throughout a person's life cycle. Galán-Rodríguez gives a very incisive definition of attachment:

Bowlby proposed a relational concept of the human being (the child enters the world primed to establish a close connection bond with a caregiver figure) supported by a very interesting conceptual framework. The contributions by Ainsworth made it possible to expand attachment theory (conceptually and academically), thus consolidating what appeared to be a productive and promising line of work (Galán-Rodríguez, 2010, p. 581).
This bond with the mother, or the attachment figure, can be decisive and will set the secure or insecure personality of the future student (Bowlby, 1969; Ainsworth \& Marvin, 1995). The child will acquire security and confidence in herself and the world, which will be very valuable when she starts school.

Another conceptual framework is the linguistic and attributive style generated by the family and social setting surrounding the child which can be encouraging or discouraging. We refer to the linguistic codes (elaborate or restricted) the child learns at home (Bernstein, 1989) and in her closest settings. Codes that then unfold - positively or negatively - in a way that is imperceptible but constant in the school.

This is where the achievement gap appears. The absence of the following factors can be decisive (National Academies of Sciences, Engineering, and Medicine, 2016, p. 81):

- A healthy life (nutrition, hygiene, sleep). - Secure attachment.

- Order, cohesion at home, predictability.

- Limits and affectionate discipline.

- Rich linguistic interaction.

- Calmness and family availability in interactions with adults.

- Educational, cultural, reading sensitivity.

Here are a few examples to illustrate the previous statements: going to public libraries with the children from an early age, joint (non-digital) play at home (Shaheen, 2014), excursions focussing on 
nature, and a belief in and support of the school from home (Tough, 2016) are in the reach of any pocket.

Therefore, fighting against the achievement gap involves: a) training families in matters such as parent-child interactions; training them in b) reinforcing key life skills (including EFs); and c) reducing stress in the lives of children and families (Center on the Developing Child at Harvard University, 2017). See Graph 1.

GRAPH 1. Three Principles to Improve Outcomes for Children and Families.

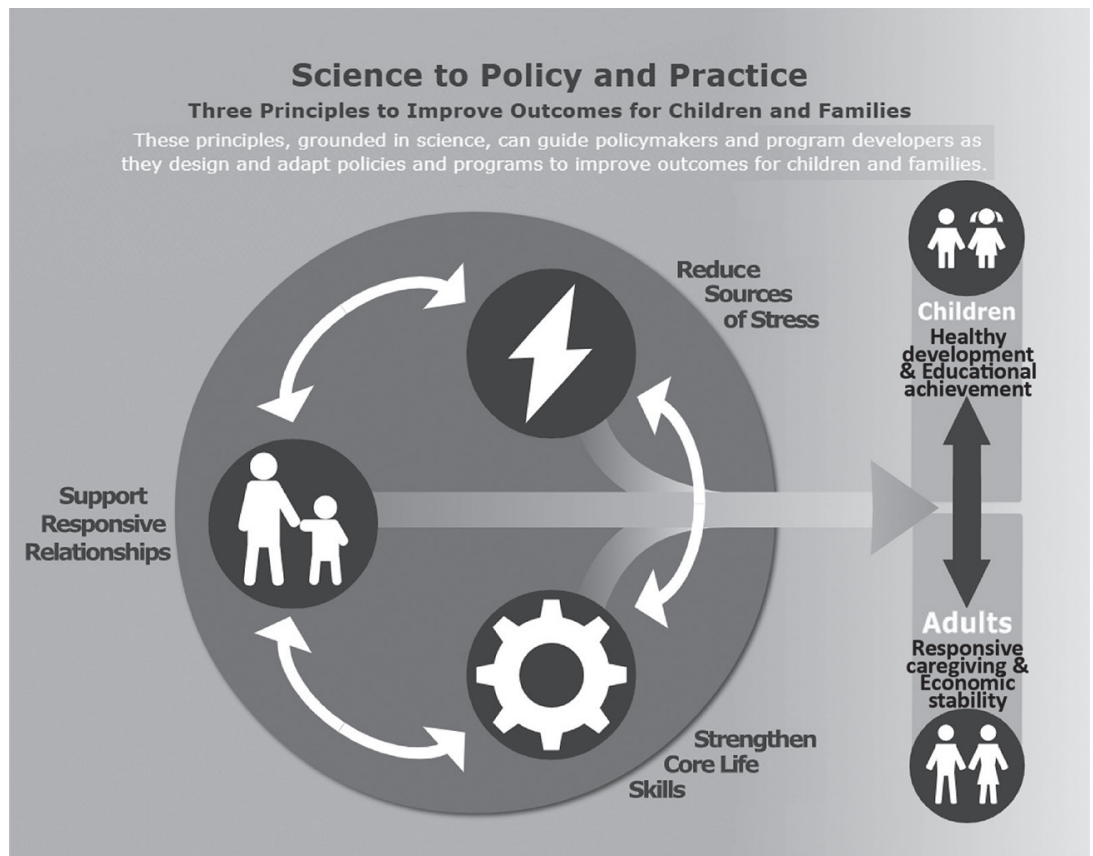

Source: Center on the Developing Child at Harvard University, 2017.

School plays a vital role but academic failure starts imperceptibly at home in the first months of life. It is again worth noting that there are families on low incomes with great sensitivity towards education and families with high incomes but very little sensitivity in this area. More than a high income, what a home needs is economic stability. And in the view of some scholars, more sobriety than opulence in their daily consumption. The social-achievement gap focusses more on a proactive family climate than on income and resources, as shown in Graph 1. However, if material resources fall below a certain poverty threshold, no family environment can overcome this adversity. Severe material deprivation is therefore a very influential stress factor.

Bourdieu (1986), a sociologist of education, has studied these structural and structuring constraints closely with his concept of habitus. Non-cognitive skills 
are part of this great humus that is habitus, established by one's social origin. This concept is considerably more complex than what is understood by habit. It comprises aesthetic and cultural perceptions, sensitivities, and inclinations. The leisure (Palmero-Cámara et al., 2015; De Bofarull, 2005) of the privileged classes also has an impact on habitus. Habitus resides in a culture that is converted into enlightened actions, choices, and attitudes. One of Bourdieu's studies is called La distinction. Critique sociale $d u$ jugement (1979). To summarise a little, it could be said that there is an ideal social-family habitus to integrate knowledge from school which is normally present in families with high levels of culture and income. At the other extreme there is a limited habitus, inclined towards being less refined, which is found in families with less prestigious cultural levels and lower incomes, which, because of its simplicity and shortcomings, does not facilitate the absorption of knowledge at school by children. The "language» of school content and subjects is often incomprehensible and distant for these latter families. Children who come from a more prestigious habitus understand the language of school perfectly.

Early childhood and primary schools sometimes work on improving these underlying conditions - restricted linguistic and cultural habitus and attachment- through the teaching and learning processes implemented, even if it is sometimes very difficult to undo them when they are deep-rooted. We reiterate that it is often the case that students lack these basic skills, and compulsory schooling assumes they will be present (Kautz, Heckman, Diris, Ter Weel, \& Borghans, 2014). This is where several variables in school failure and dropout can be found: in families' internal attitudes, beliefs, perceptions, and involvement with children at an early age. The influential PISA reports have, since 2012, started considering the existence of these skills as part of their task of evaluating 15-year-old students in OECD countries: Students' Engagement, Drive and Self-Beliefs (OECD, 2013). And this is perhaps the first step towards different countries regarding the student not as an abstract being who learns but as a complex, diverse person with different needs and backgrounds in different areas: cognitive, psychological, social, and material. These material needs often combine with acute and stressful adversities (unemployment, lack of job security, illness, single-parent families, etc.) that have not just grown but multiplied in recent years given the instability - political, economic, workplace, family - the West has been experiencing since the 2008 crisis.

\section{Social and emotional learning and self-regulation}

In this direction, for several years schools have been promoting education in values that can overcome shortcomings in the social, cultural, educational, and emotional areas that specific students bring from their social-family environment and that can hold back the students' academic development. In recent years, education in values has been 
developed using a solid framework from the English-speaking world: social and emotional learning (SEL). Social and emotional learning (Durlak, Dymnicki, Taylor, Weissberg, \& Schellinger, 2011; Álvarez-Hevia, 2018) is an umbrella term that once again includes skills that go beyond classical intelligence and which help to lay the foundations for learning from different personality features.

Social and emotional learning is an important step forward in moral education; it helps improve the atmosphere in class and at school and its results can be measured using the Big Five questionnaire mentioned above (John \& Srivatava, 1999). Social and emotional learning comprises five core competences: 1 ) self-awareness; 2) self-management; 3) social awareness; 4) relationship skills; and 5) responsible decision-making. It has proved very effective in the USA and has had a very good cost-benefit ratio (1:11 per dollar invested) when applied in schooling (Elias et al., 2015). Nonetheless, it is very important to note that the application of this conceptual framework requires systematic integration where a) the home and community, b) the school, and c) the class play an interdependent role. The development of character, habits, and self-regulation is only possible if these educational agents converge. In other words, a calm, opportune, and organised social and emotional learning can only grow from these competences. See Table 2 and Graph 2. We will allow them to define themselves and will note the points that overlap with the objectives of this study:
Social and emotional learning (SEL) is the process by which children and adults acquire and correctly apply the necessary knowledge, attitudes, and skills for understanding and managing emotions, setting and achieving positive goals, feeling and showing empathy for others, establishing and maintaining positive relationships, and taking responsible decisions (CASEL, 2017, p. 3).

Emotional intelligence will also be the basis of more reflexive and ethical decisions at school and in life.

\section{The big five: personality dimensions}

Non-cognitive intelligence and its close relative emotional intelligence, based on character skills and the cultivation of cognitive-behavioural habits, therefore comprise a broad spectrum of competences, skills, and strengths that must work together to direct the cognition-deliberation-decision-action process. The present study focusses on the worlds of school and family (although it has other applications: professional, occupational, community, etc.). These competences and habits must be integrated to achieve results as they do not work if there are just some of them but not others. People advance in character skills if they progress in most areas of each one of them. The Big Five questionnaire measures these strengths and detects shortcomings in them in five personality factors, from different perspectives and in their respective aspects (Table 3). Heckman and Kautz (2012) provide an approximate outline of what non-cognitive skills are and their effects. 
TABLE 2. Conceptual framework for systematic social and emotional learning.

\begin{tabular}{|l|l|l|}
$\begin{array}{l}\text { Homes and community } \\
\text { associated with the } \\
\text { school }\end{array}$ & Self-management & $\begin{array}{l}\text { Self-discipline } \\
\text { Self-motivation } \\
\text { Goal-setting } \\
\text { Organizational skills }\end{array}$ \\
\cline { 2 - 3 } \begin{tabular}{l} 
Schools with SEL \\
practices in all areas \\
\multicolumn{1}{c|}{+}
\end{tabular} & $\begin{array}{l}\text { Responsible } \\
\text { decision making } \\
\text { Classes where SEL } \\
\text { is applied in the curric- } \\
\text { ulum }\end{array}$ & $\begin{array}{l}\text { Identifying problems } \\
\text { Analysing situations } \\
\text { Solving problems } \\
\text { Evaluating } \\
\text { Reflecting } \\
\text { Ethical responsibility }\end{array}$ \\
\cline { 2 - 3 } & Relationship skills & $\begin{array}{l}\text { Communication } \\
\text { Social engagement } \\
\text { Relationship building } \\
\text { Team work }\end{array}$ \\
& & $\begin{array}{l}\text { Perspective taking } \\
\text { Empathy } \\
\text { Appreciating diversity } \\
\text { Respect for others }\end{array}$ \\
\cline { 2 - 3 } & Social awareness &
\end{tabular}

Source: CASEL, 2017, p. 3.

Identifying emotions

Accurate self-perception

Recognizing strengths

Self-confidence

Self-efficacy

Impulse control

Stress management

Self-discipline

Self-motivation

Goal-setting

Organizational skills

Identifying problems

Analysing situations

Solving problems

Evaluating

Reflecting

Communication

Social engagement

Relationship building

Perspective taking

Empathy

Respect for others

GrapH 2. Systemic Social and Emotional Learning.

Source: Casel, 2017, p. 3.

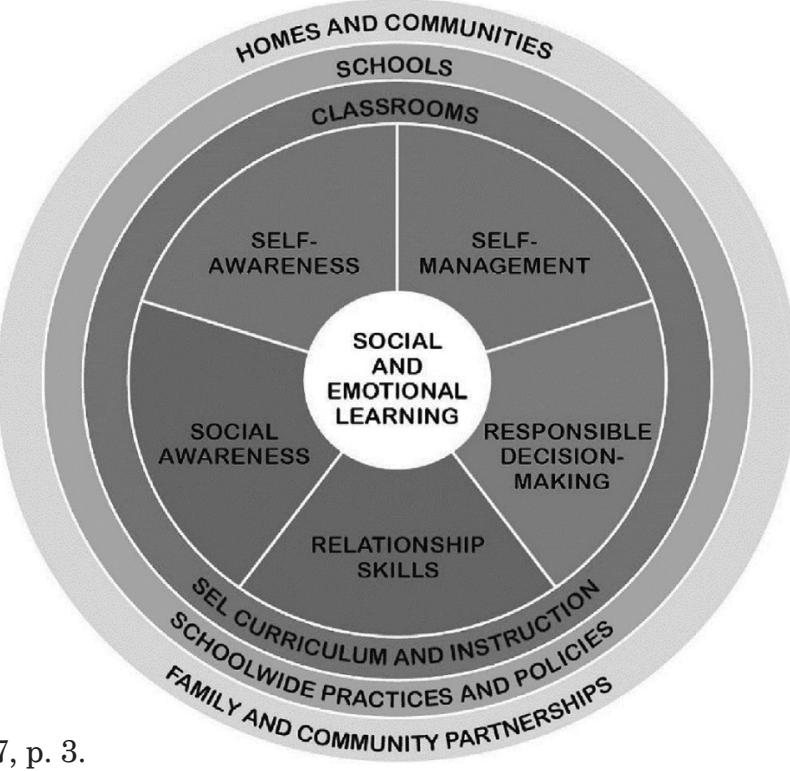


These researchers have shown that students (and workers, etc.) who have these skills display high performance levels at school and at work, not just in the technical-academic area but also in a good and decisive character that can manage challenges and difficulties, both material and relational based on reasonable emo- tional stability. This table gives an overview of the main personality traits that are so closely related to character skills, to non-cognitive skills. The table below provides a brief (and limited) definition of these fields and at the same time helps us understand their study, implementation, and evaluation. Table 3.

TABLE 3. The Big Five Domains and their facets.

\begin{tabular}{|c|c|c|c|c|}
\hline $\begin{array}{c}\text { Big Five } \\
\text { Personality } \\
\text { Factors }\end{array}$ & $\begin{array}{l}\text { American } \\
\text { Psychology } \\
\text { Association } \\
\text { Dictionary } \\
\text { Description } 1\end{array}$ & $\begin{array}{l}\text { Facets (and } \\
\text { correlated } \\
\text { trait-adjective) }\end{array}$ & Related traits & $\begin{array}{l}\text { Analogous } \\
\text { childhood } \\
\text { temperament } \\
\text { traits }\end{array}$ \\
\hline $\begin{array}{l}\text { Openness to } \\
\text { experience }\end{array}$ & $\begin{array}{l}\text { «The tendency to } \\
\text { be open to new } \\
\text { aesthetic, cultur- } \\
\text { al, or intellectual } \\
\text { experiences». }\end{array}$ & $\begin{array}{l}\text { Fantasy (imagi- } \\
\text { native); aesthetic } \\
\text { (artistic); feelings } \\
\text { (excitable); } \\
\text { actions (wide } \\
\text { interests); ideas } \\
\text { (curious); and } \\
\text { values (uncon- } \\
\text { ventional). }\end{array}$ & & $\begin{array}{l}\text { Sensory sensitiv- } \\
\text { ity; pleasure in } \\
\text { low-intensity ac- } \\
\text { tivities; curiosity. }\end{array}$ \\
\hline $\begin{array}{l}\text { Conscien- } \\
\text { tiousness }\end{array}$ & $\begin{array}{l}\text { «The tendency } \\
\text { to be organized, } \\
\text { responsible, and } \\
\text { hardworking». }\end{array}$ & $\begin{array}{l}\text { Competence } \\
\text { (efficient); order } \\
\text { (organized); } \\
\text { dutifulness } \\
\text { (not careless); } \\
\text { achievement } \\
\text { striving (ambi- } \\
\text { tious); self-disci- } \\
\text { pline (not lazy); } \\
\text { and deliberation } \\
\text { (not impulsive). }\end{array}$ & $\begin{array}{l}\text { Grit, persever- } \\
\text { ance, delay of } \\
\text { gratification, } \\
\text { impulse control, } \\
\text { achievement } \\
\text { striving, ambi- } \\
\text { tion, and work } \\
\text { ethic. }\end{array}$ & $\begin{array}{l}\text { Attention (lack } \\
\text { of); distracti- } \\
\text { bility; effortful } \\
\text { control; impulse } \\
\text { control/delay of } \\
\text { gratification; per- } \\
\text { sistence; activity. }\end{array}$ \\
\hline
\end{tabular}




\begin{tabular}{|c|c|c|c|c|}
\hline Extroversion & $\begin{array}{l}\text { «An orientation } \\
\text { of one's interests } \\
\text { and energies } \\
\text { toward the outer } \\
\text { world of people } \\
\text { and things rather } \\
\text { than the inner } \\
\text { world of subject- } \\
\text { ive experience; } \\
\text { characterized by } \\
\text { positive affect } \\
\text { and sociability». }\end{array}$ & $\begin{array}{l}\text { Warmth (friend- } \\
\text { ly); gregarious- } \\
\text { ness (sociable); } \\
\text { assertiveness } \\
\text { (self-confident); } \\
\text { activity (energet- } \\
\text { ic); excitement } \\
\text { seeking (adven- } \\
\text { turous); and } \\
\text { positive emotions } \\
\text { (enthusiastic). }\end{array}$ & & $\begin{array}{l}\text { Surgency; social } \\
\text { dominance; } \\
\text { social vitali- } \\
\text { ty; sensation } \\
\text { seeking; shyness; } \\
\text { activity; positive } \\
\text { emotionality; and } \\
\text { sociability/affili- } \\
\text { ation. }\end{array}$ \\
\hline Agreeableness & $\begin{array}{l}\text { «The tendency to } \\
\text { act in a cooper- } \\
\text { ative, unselfish } \\
\text { manner». }\end{array}$ & $\begin{array}{l}\text { Trust (forgiving); } \\
\text { straightfor- } \\
\text { wardness (not } \\
\text { demanding); } \\
\text { altruism (warm); } \\
\text { compliance (not } \\
\text { stubborn); mod- } \\
\text { esty (not show- } \\
\text { off); and Ten- } \\
\text { der-mindedness } \\
\text { (sympathetic). }\end{array}$ & $\begin{array}{l}\text { Empathy; per- } \\
\text { spective taking; } \\
\text { cooperation; and } \\
\text { competitiveness. }\end{array}$ & $\begin{array}{l}\text { Irritability; ag- } \\
\text { gressiveness; and } \\
\text { wilfulness. }\end{array}$ \\
\hline $\begin{array}{l}\text { Neuroticism/ } \\
\text { Emotional } \\
\text { Stability }\end{array}$ & $\begin{array}{l}\text { Emotional } \\
\text { stability is } \\
\text { «predictability } \\
\text { and consistency } \\
\text { in emotional } \\
\text { reactions, with } \\
\text { absence of rapid } \\
\text { mood changes». } \\
\text { Neuroticism is } \\
\text { «a chronic level } \\
\text { of emotional } \\
\text { instability and } \\
\text { proneness to } \\
\text { psychological } \\
\text { distress». }\end{array}$ & $\begin{array}{l}\text { Anxiety (wor- } \\
\text { rying); hostil- } \\
\text { ity (irritable); } \\
\text { depression (not } \\
\text { contented), } \\
\text { self-conscious- } \\
\text { ness (shy); } \\
\text { impulsiveness } \\
\text { (moody); vulner- } \\
\text { ability to stress } \\
\text { (not self-confi- } \\
\text { dent). }\end{array}$ & $\begin{array}{l}\text { Internal vs. } \\
\text { external; locus } \\
\text { of control; core } \\
\text { self-evaluation; } \\
\text { self-esteem; } \\
\text { self-efficacy; op- } \\
\text { timism; and Axis } \\
\text { I psychopatholo- } \\
\text { gies (mental dis- } \\
\text { orders) including } \\
\text { depression and } \\
\text { anxiety disor- } \\
\text { ders. }\end{array}$ & $\begin{array}{l}\text { Fearfulness/ } \\
\text { behavioural inhi- } \\
\text { bition; Shyness; } \\
\text { Irritability; Frus- } \\
\text { tration; (lack of } \\
\text { ) soothability; } \\
\text { sadness. }\end{array}$ \\
\hline
\end{tabular}

Source: Adapted from Heckman \& Kautz, 2012 and John \& Srivastava, 1999. 


\section{Conclusion: learning to think in a more complex future}

To summarise, in the world of education it has been apparent for years that it is not enough to have cognitive intelligence (measured by the intelligence quotient), but that it is also vital to emphasise the non-cognitive intelligence that must form its foundation: character skills. These character skills will consequently be present all through life: in secondary school, in higher education, in professional life, in social life, and also as the basis of a stable family life.

Research from the English-speaking world has found that character skills are the basis of human capital (the skills and capacities that create value in the professional world and lead to individual improvements for employers, businesses, a country's productivity, etc.) (Heckman, 2011). But character, good character is also the basis of civic life understood as the result of various aspects working in concordance: the consistency of family life (which always has social returns); participation by citizens; civic virtues; the absence of crime; and its members overcoming psycho-social pathologies (Nucci, Krettenauer, \& Narváez, 2014).

We are in the early years of a century which is starting with challenges and increasing complexity in many fields: a new era - the fourth industrial revolutionthat demands a new education for a new type of citizen in a changing world. This century requires adaptable and skilled personalities who can manage complexity. Accordingly, many experts speak of 21st Century skills (National Research Council, 2012) to emphasise levels of operative intelligence that are as central as critical thinking, decision-making, and problem-solving skills in the line of executive functions. These skills emphasise the competence-based focus and are in demand from international organisations such as the OECD (Ananiadou \& Claro, 2009). It is no longer enough to cultivate strategic skills but new citizens must also be equipped with decisive internal attitudes, agile mentalities, and flexible world views.

The mindset — attitudes and beliefsis therefore embedded in character skills. This is the field of interior convictions, attributive styles, and psychological tone that can either encourage work and intrinsic motivation or can discourage and paralyse. The self-determination theory of Deci and Ryan (2000) who have studied intrinsic motivation fits into this sphere very well. It is also the field of self-efficacy as studied by Bandura (1997). Children, students, and future citizens must incorporate visions of themselves and of the world that enable them to gain in competence, autonomy, and relational capacity without being intoxicated by a sometimes alienating and destructive setting.

Some areas of content from digital media can have a negative effect and also have a distracting and anxiogenic effect (Hoge, Bickham, \& Cantor, 2017) through various devices, especially through excessive use of mobile phones and social media. Distractions, lack of attention, and 
lack of concentration in the classroom and when studying are ever more acute difficulties education must confront (Scherer \& Hatlevik, 2017).

Nonetheless, the prospects for change are encouraging. This is a teachable field. One good example is the studies into passion and perseverance —or grit- carried out by Duckworth (2013). This is a broad topic with different ramifications we cannot consider here. The need for silence, the ability to calm oneself, the ability to enter into the interior of the self to concentrate, be fully attentive, and channel stress could also be included. Our students can be highly stressed, even when very young, and it is important that they attain the stabilising rest that favours learning as Kabat-Zinn (2003) has studied in the broad sphere of mindfulness. Character, attitude, resilience, and inner peace are the preconditions for the best educational attainment. In these final lines we consider psychological stability as one of the foundations of learning that results in overcoming anxiety, fear, sadness, low self-concept, and continuous change in emotional stability. Part of this field can again be measured by the Big Five Questionnaire (BFQ) in its neuroticism/emotional stability personality factor.

The main aim of this work is to put the importance of character intelligence at the centre of the educational agenda. It is necessary to invite educational agents into the debate about these topics. We believe that character intelligence is somewhat neglected; it is not just moral training but also intellectual training. The first step towards promoting character skills is to establish whether they really influence educational achievement and emotional wellbeing. The corpus of research into this topic is small but promising (Khine \& Areepattamannil, 2016; Roberts et al., 2015). In conclusion, there are various measurement instruments in this field Big Three, MPQ, Big Nine- but in here we have proposed one of the most comprehensive and thoroughly tested: the Big Five Questionnaire in its version for schools and for the Hispanic world, the $\mathrm{BFQ}-\mathrm{N}$ mentioned above. We also have the BRIEF-2 instrument, also validated for the Spanish-speaking world and for early-years/primary use. BRIEF-2 is intended to evaluate EFs (executive functions) that we believe are at the core of character skills. This triangulation that opens doors for the voices of families as BRIEF-2 has one version for teachers and another for parents.

\section{Notes}

${ }^{1}$ These definitions come from the Dictionary of the American Psychological Association (VandenBos, 2015).

\section{References}

Ainsworth, M. D., \& Marvin, R. S. (1995). On the shaping of attachment theory and research: An interview with Mary DS Ainsworth (Fall 1994). Monographs of the society for research in child development, 60 (2-3), 3-21. doi: http://dx.doi. org $/ 10.2307 / 1166167$

Alcover, C., \& Rodríguez, F. (2012). Plasticidad Cerebral y Hábito en William James: un Antecedente para la Neurociencia Social. Psychologia Latina, 3 (1), 1-9. 
Althof, W., \& Berkowitz, M. W. (2006). Moral education and character education: Their relationship and roles in citizenship education. Journal of Moral Education, 35 (4), 495-518. doi: https://doi.org/10.1080/03057240601012204

Álvarez-Hevia, D. M. (2018). Aproximación crítica a la Inteligencia Emocional como discurso dominante en el ámbito educativo. revista española de pedagogía, 76 (269), 7-23. doi: https://doi.org/10.22550/REP76-1-2018-01

Ananiadou, K., \& Claro, M. (2009). $21^{\text {st }}$ Century Skills and Competences for New Millennium Learners in OECD Countries. OECD Education Working Papers, 41. doi: https://doi. org $/ 10.1787 / 19939019$

Bandura, A. (1977). Self-efficacy: toward a unifying theory of behavioral change. Psychological review, 84 (2), 191-215. doi: https://doi. org/10.1016/0146-6402(78)90002-4

Bernacer, J., \& Murillo, J. I. (2014). The Aristotelian conception of habit and its contribution to human neuroscience. Frontiers in human neuroscience, 8, 1-10. doi: https://doi. org/10.3389/fnhum.2014.00883

Bernstein, B. (1989). Clases, códigos y control (Vol. 1). Madrid: Ediciones Akal.

Bourdieu, P. (1979). La distinction. Critique sociale du jugement. Paris: Minuit.

Bourdieu, P. (1986). Habitus, code et codification. Actes de la recherche en sciences sociales, 64 (1), 40-44. doi: https://doi.org/10.3406/ arss. 1986.2335

Bowlby, J. (1969). Attachment and loss: Vol. 1. Attachment. New York: Basic Books.

Bowlby, J. (1973). Attachment and loss: Vol. 2. Separation: Anxiety and anger. New York: Basic Books.

Bronfenbrenner, U. (2009). The ecology of human development. Cambridge: Harvard University Press.

CASEL (2017). Educating Hearts, Inspiring Minds. Core SEL competencies. Retrieved from https://casel.org/core-competencies/ (Consulted on $15 / 01 / 2018)$.

Carrasco-Ortiz, M. Á., Holgado-Tello, F. P., \& Del Barrio-Gandara, M. V. (2005). Dimensionalidad del cuestionario de los cinco grandes (BFQ-N) en población infantil española. Psicothema, 17 (2), 286-291.

Center on the Developing Child at Harvard University (2017). Three Principles to Improve Outcomes for Children and Families. Retrieved from https://bit.ly/2gSMqh2 (Consulted on 20/02/2018).

Chen, Q., \& Yan, Z. (2016). Does multitasking with mobile phones affect learning? A review. Computers in Human Behavior, 54, 34-42. doi: https://doi.org/10.1016/j.chb.2015.07.047

Damasio, A. R. (1996). El error de Descartes. Barcelona: Crítica.

De Bofarull, I. (2005). Ocio y tiempo libre: un reto para la familia. Pamplona: Eunsa.

De Bofarull, I., \& Coma, M. A. (2017). Possibilities and limitations of digital multitasking for school homework. Quaderns de Polítiques Familiars, 3, 48-58.

Diamond, A. (2013). Executive functions. Annual review of psychology, 64, 135-168. doi: https:// doi.org/10.1146/annurevpsych-113011-143750

Diamond, A. (2014). Want to optimize executive functions and academic outcomes? Simple, just nourish the human spirit. Minnesota Symposia on Child Psychology, 37, 205-232.

Diamond, A., \& Ay Lee, K. (2011). Interventions shown to aid executive function development in children 4 to 12 years old. Science, 333 (6045), 959-964. doi: https://doi.org/10.1126/ science. 1204529

Duckworth, A. L. (2013). True grit. The Observer, $26(4), 1-3$.

Durlak, J. A., Dymnicki, A. B., Taylor, R., Weissberg, R. P., \& Schellinger, K. B. (2011). The impact of enhancing students' social and emotional learning: A meta-analysis of school-based universal interventions. Child Development, 82, 405-432.

Dweck, C. S. (2006). Mindset: The new psychology of success. New York: Random House Incorporated.

Elias, M. J., Leverett, L., Duffell, J. C., Humphrey, N., Stepney, C., \& Ferrito, J. (2015). Integrating SEL with related prevention and youth development approaches. In J. A. Durlak, C. E. Domitrovich, R. P. Weissberg, \& T. P. Gullotta (Eds.), Handbook for social and emotional learning: 
Research and practice (pp. 33-49). New York: Guilford.

Galán-Rodríguez, A. (2010). El apego: Más allá de un concepto inspirador. Revista de la Asociación Espavñola de Neuropsiquiatría, 30 (4), 581-595.

Gervilla, E. (2000). Un modelo axiológico de educación integral. revista española de pedagogía, 58 (215), 39-57.

Gioia, G. A., Isquith, P. K., Guy, S. C., \& Kenworthy, L. (2000). Test review behavior rating inventory of executive function. Child Neuropsychology, 6 (3), 235-238.

Hanscombe, K. B., Haworth, C. M., Davis, O. S., Jaffee, S. R., \& Plomin, R. (2011). Chaotic homes and school achievement: a twin study. Journal of Child Psychology and Psychiatry, and Allied Disciplines, 52 (11), 1212-1220. doi: https://doi. org/10.1111/j.1469-7610.2011.02421.x

Heckman, J. J., (2011). The Economics of Inequality: The Value of Early Childhood Education. American Educator, 35 (1), 31-35.

Heckman, J. J., \& Kautz, T. (2012). Hard evidence on soft skills. Labour economics, 19 (4), 451464.

Heckman, J. J., \& Kautz, T. (2013). Fostering and Measuring Skills: Interventions That Improve Character and Cognition. National Bureau of Economic Research, 19656. doi: https://doi. org/10.3386/w19656

Hoge, E., Bickham, D., \& Cantor, J. (2017). Digital media, anxiety, and depression in children. Pediatrics, 140 (2), S76-S80. doi: https://doi. org/10.1542/peds.2016-1758G

James, W. (1899). Talks to Teachers on Psychology and to Students on Some of Life's Ideals. Boston: Harvard University Press.

Jennings, P. A., \& Greenberg, M. T. (2009). The prosocial classroom: Teacher social and emotional competence in relation to student and classroom outcomes. Review of Educational Research, 79 (1), 491-525.

John, O. P., \& Srivastava, S. (1999). The Big Five trait taxonomy: History, measurement, and theoretical perspectives. In L. A. Pervin, \& 0 . P. John (Eds.), Handbook of personality: Theory and research (pp. 102-138). New York: Guilford Press.
Kabat-Zinn, J. (2003). Mindfulness-based interventions in context: past, present, and future. Clinical psychology. Science and practice, 10 (2), 144-156.

Kautz, T., Heckman, J. J., Diris, R., Ter Weel, B., \& Borghans, L. (2014). Fostering and measuring skills: Improving cognitive and non-cognitive skills to promote lifetime success. National Bureau of Economic Research, 20749, 1-118. doi: https://doi.org/10.3386/w20749

Khine, M. S., \& Areepattamannil, S. (Eds.) (2016). Non-cognitive Skills and Factors in Educational Attainment. Rotterdam: Sense Publishers.

Lickona, T. (1991). Educating for character: How our schools can teach respect and responsibility. New York: Bantam Books.

Maldonado-Belmonte, M. J. (2016). Adaptación del BRIEF (Behavior Rating Inventory of Executive Function) a población española y su utilidad para el diagnóstico del trastorno por déficit de atención-hiperactividad subtipos inatento y combinado (Doctoral thesis). Universidad Complutense de Madrid. Retrieved from http://eprints.ucm.es/37563/1/T37182.pdf (Consulted on 14/02/2018).

Malikail, J. (2003). Moral character: hexis, habitus and 'habit'. An Internet Journal of Philosophy, 7, 1-22.

McEwen, B. S. (2016). In pursuit of resilience: stress, epigenetics, and brain plasticity. Annals of the New York Academy of Sciences, 1373 (1), 56-64.

Moullin, S., Waldfogel, J., \& Washbrook, E. (2014). Baby Bonds: Parenting, attachment and a secure base for children. The Sutton Trust. Retrieved from http://dera.ioe.ac.uk/30276/1/baby-bonds-final-1.pdf (Consulted on 24/10/2018).

National Academies of Sciences, Engineering, and Medicine (2016). Parenting matters: Supporting parents of children ages 0-8. Washington, D.C.: The National Academies Press.

National Research Council (2012). Education for life and work: Developing transferable knowledge and skills for the 21st century. Washington, D.C.: The National Academies Press.

Nucci, L., Krettenauer, T., \& Narváez, D. (Eds.) (2014). Handbook of moral and character education. New York: Routledge. 
OECD (2013). PISA 2012 Results: Ready to Learn (Volume III): Students' Engagement, Drive and Self-Beliefs. Paris: OECD Publishing.

Palmero-Cámara, C., Jiménez-Martín, J., \& Jiménez-Eguizábal, A. (2015). Ocio, Política y Educación. Reflexiones y retos veinticinco siglos después de Aristóteles. revista española de pedagogía, 73 (216), 5-21.

Perry, B. D. (2009). Examining child maltreatment through a neurodevelopmental lens: Clinical applications of the neurosequential model of therapeutics. Journal of Loss and Trauma, 14 (4), 240-255.

Real Academia Española (2017). Diccionario de la lengua española (23.1 ed.). Retrieved from http://www.rae.es/ (Consulted on 25/10/18).

Roberts, R. D., Martin, J., \& Olaru, G. (2015). A Rosetta Stone for noncognitive skills: Understanding, assessing, and enhancing noncognitive skills in primary and secondary education. New York: Asia Society and ProExam.

Ryan, R. M., \& Deci, E. L. (2000). Intrinsic and extrinsic motivations: Classic definitions and new directions. Contemporary educational psychology, 25 (1), 54-67.

Scherer, R., \& Hatlevik, O. E. (2017). «Sore eyes and distracted» or «excited and confident»?-The role of perceived negative consequences of using ICT for perceived usefulness and self-efficacy. Computers \& Education, 115, 188-200. doi: https://doi.org/10.1016/j. compedu.2017.08.003

Shaheen, S. (2014). How child's play impacts executive function-related behaviors. Applied Neuropsychology: Child, 3 (3), 182-187. doi: https:// doi.org/10.1080/21622965.2013.839612

Shonkoff, J. P., Boyce, W. T., \& McEwen, B. S. (2009). Neuroscience, molecular biology, and the childhood roots of health disparities: building a new framework for health promotion and disease prevention. Jama, 301 (21), 2252-2259. doi: https://doi.org/10.1001/jama.2009.754

Soprano, A. M. (2003). Evaluación de las funciones ejecutivas en el niño. Revista de neurología, 37 (1), 44-50.

Taylor, R. D., Oberle, E., Durlak, J. A., \& Weissberg, R. P. (2017). Promoting Positive Youth
Development Through School-Based Social and Emotional Learning Interventions: A Meta-Analysis of Follow-Up Effects. Child development, 88 (4), 1156-1171. doi: https://doi. org/10.1111/cdev. 12864

Thoilliez, B. (2013). El pensamiento pedagógico de William James: el tactful teacher y la educación moral. Foro de Educación, 11 (15), 83-102. doi: http://dx.doi.org/10.14516/ fde.2013.011.015.004

Tough, P. (2014). Cómo triunfan los niños: Determinación, curiosidad y el poder del carácter. Madrid: Palabra.

Tough, P. (2016). Helping children succeed: What works and why. New York: Random House.

VandenBos, G. R. (Ed.) (2015). American Psychological Association. APA Dictionary of Psychology. Washington: American Psychological Association.

Vernon-Feagans, L., Willoughby, M., \& Garrett-Peters, P. (2016). Predictors of behavioral regulation in kindergarten: Household chaos, parenting, and early executive functions. Developmental psychology, 52 (3), 430441.

Vigo, A. G. (2012). Deliberación y decisión según Aristóteles. Tópicos, 43, 51-92.

West, M. R., Kraft, M. A., Finn, A. S., Martin, R. E., Duckworth, A. L., Gabrieli, C. F., \& Gabrieli, J. D. (2016). Promise and paradox: Measuring students' non-cognitive skills and the impact of schooling. Educational Evaluation and Policy Analysis, 38 (1), 148-170. doi: https://doi. org/10.3102/0162373715597298

\section{Author's biography}

Ignasi de Bofarull holds a degree in Geography and History from the Universitat Autónoma de Barcelona and $\mathrm{a} \mathrm{PhD}$ in Humanities from the Universitat Internacional de Catalunya. He currently holds the positions of Associate Professor of Family Sociology and Education at the UIC Faculty of Education, Researcher at the UIC Institute 
for Advanced Studies of the Family and Director of the research group of the Generalitat de Catalunya called Parentalitat, Igualtat i Conciliació.

(iD https://orcid.org/0000-0003-4896-1997

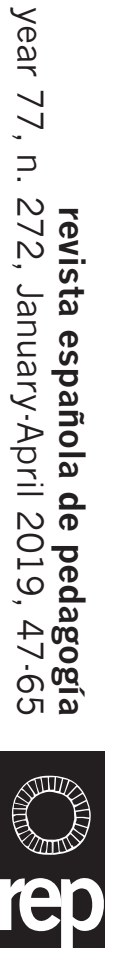




\section{revista española de pedagogía año 77, n 272, enero-abril 2019 \\ Spanish Journal of Pedagogy year 77, n. 272, January-April 2019 \\ Table of Contents \\ Sumario}

\section{Studies}

\section{Estudios}

Francisco López Rupérez, Isabel García García,

\section{Eva Expósito Casas}

Performance in science, epistemic conceptions, and STEM vocations in Spain's autonomous communities: evidence from PISA 2015, improvement policies, and practices Rendimiento en Ciencias, concepciones epistémicas y vocaciones STEM en las comunidades autónomas españolas. Evidencias desde PISA 2015, políticas y prácticas de mejora $\mathbf{5}$

\section{Zaida Espinosa Zárate}

Cultivating creativity for intercultural dialogue

El cultivo de la creatividad para el diálogo intercultural

Ignasi de Bofarull

Character and learning habits:

definition and measurement proposal

Carácter y hábitos para el aprendizaje:

definición y proyecto de medición

David Luque

Interpretive developments of the philosophy of education in the anglophone tradition: an attempt to systematise them Desarrollos interpretativos de la filosofía de la educación en la tradición anglófona: un intento de sistematización

\section{Notes}

Notas

María Moralo, Manuel Montanero

Learning with and without errors in students with ASD Aprendizaje con y sin error en estudiantes con TEA

Antonio Portela Pruaño, José Miguel Nieto Cano, Ana Torres Soto

Re-engagement in education and training of young people who leave education early: the importance of earlier prior trajectories

La reincorporación formativa de jóvenes

que abandonan tempranamente la educación:

relevancia de su trayectoria previa

Oihane Fernández-Lasarte, Eider Goñi, Igor Camino,

Estibaliz Ramos-Díaz

Perceived social support and school engagement in secondary students

47 Apoyo social percibido e implicación escolar del alumnado de educación secundaria

Jesús-Miguel Muñoz-Cantero, Luisa Losada-Puente Validation of the construct of self-determination through the ARC.INICO scale for teenagers Validación del constructo de autodeterminación a través de la escala ARC.INICO para adolescentes 


\section{Månmaculada López-Núñez, Susana Rubio-Valdehita,}

Eva Ma Diaz-Ramiro

Changes in Psychology students' workload due to

alignment with the EHE

Cambios producidos por la adaptación al EEES

en la carga de trabajo de estudiantes de Psicología

\section{Book reviews}

Naval, C., \& Arbués, E. (Eds.). Hacer la Universidad

en el espacio social [Constructing the university in the

social space] (Emanuele Balduzzi). Mínguez, R., \&

Romero, E. (Coords.). La educación ciudadana en

un mundo en transformación: miradas y propuestas

[Citizenship education in a changing world:

perspectives and proposals] (Marina Pedreño Plana).

Bellamy, F.-X. Los desheredados: por qué es urgente

transmitir la cultura [The disinherited: why transmitting

culture is urgent] (Esteban López.Escobar). Pallarés,

M., Chiva, O., López Martín, R., \& Cabero, I. La

escuela que llega. Tendencias y nuevos enfoques

metodológicos [The school of the future: Trends and

new methodological focuses] (Juan Carlos San Pedro

Velado).

This is the English version of the research articles and book reviews published originally in the Spanish printed version of issue 272 of the revista española de pedagogía. The full Spanish version of this issue can also be found on the journal's website http://revistadepedagogia.org.

ISSN: 0034-9461 (Print), 2174-0909 (Online)

https://revistadepedagogia.org/

Depósito legal: M. 6.020 - 1958 